\title{
Relationship between embedding-potential eigenvalues and topological invariants of time-reversal invariant band insulators
}

\author{
H. Ishida ${ }^{1,2}$ and D. Wortmann ${ }^{3}$ \\ ${ }^{1}$ College of Humanities and Sciences, Nihon University, Tokyo 156-8550, Japan \\ ${ }^{2}$ Center for Materials Research by Information Integration, National Institute for Materials Science, Tsukuba, Ibaraki 305-0047, Japan \\ ${ }^{3}$ Peter Grünberg Institut and Institute of Advanced Simulations, Forschungszentrum Jülich, 52425 Jülich, Germany \\ (Received 28 December 2015; revised manuscript received 19 February 2016; published 9 March 2016)
}

\begin{abstract}
The embedding potential defined on the boundary surface of a semi-infinite crystal relates the value and normal derivative of generalized Bloch states propagating or decaying toward the interior of the crystal. It becomes Hermitian when the electron energy $\epsilon$ is located in a projected bulk band gap at a given wave vector $\mathbf{k}$ in the surface Brillouin zone (SBZ). If one plots the real eigenvalues of the embedding potential for a time-reversal invariant insulator in the projected bulk band gap along a path $\epsilon=\epsilon_{0}(\mathbf{k})$ passing between two time-reversal invariant momentum (TRIM) points in the SBZ, then, they form Kramers doublets at both end points. We will demonstrate that the $Z_{2}$ topological invariant, $v$, which is either 0 or 1 , depending on the product of time-reversal polarizations at the two TRIM points, can be determined from the two different ways these eigenvalues are connected between the two TRIM points. Furthermore, we will reveal a relation, $v=P$ mod 2, where $P$ denotes the number of poles that the embedding potential exhibits along the path. We also discuss why gapless surface states crossing the bulk band gap inevitably occur on the surface of topological band insulators from the view point of the embedding theory.
\end{abstract}

DOI: 10.1103/PhysRevB.93.115415

\section{INTRODUCTION}

Since the discovery that band insulators with time-reversal symmetry can be divided according to $Z_{2}$ topological invariants characterizing their electronic band structure, there has been a continuing effort to search for topological insulators [1-4]. A salient feature of topological insulators is the presence of time-reversal protected gapless surface (edge) bands crossing the energy gap between the valence and conduction bands $[5,6]$. Their energy dispersion relation with wave vector possesses the same form as that of massless Dirac fermions in the vicinity of a time-reversal invariant momentum (TRIM) in the surface Brillouin zone (SBZ). To utilize surface conduction through massless Dirac bands exhibiting chiral spin texture for technological applications such as spintronics, it is desirable to have materials with a large band gap in order to reduce the influence of bulk electron conduction. On the other hand, the energy-gap values of topological band insulators known to date are not so large, since they become topologically nontrivial via the band inversion mechanism associated with large spin-orbit interactions. Among three-dimensional (3D) topological band insulators with relatively large band gaps are binary and ternary chalcogenide compounds containing heavy elements such as $\mathrm{Bi}_{2} \mathrm{Se}_{3}$ and $\mathrm{TlBiSe} \mathrm{B}_{2}$ whose bulk band gap amounts to a few tenths of eV [7-15]. It is remarkable that these compounds were at first theoretically predicted to be topological insulators before experimental verifications $[7,11]$.

One can define a single $Z_{2}$ invariant for two-dimensional (2D) band insulators with time-reversal symmetry [16,17], whereas there are four $Z_{2}$ invariants $\left(v_{0} ; v_{1} v_{2} v_{3}\right)$ for the 3D ones [18-21]. As shown by Fu and Kane [20], the $Z_{2}$ invariants of 2D (3D) band insulators having a center of space inversion can be easily calculated from the parities of valence-band wave functions at the four (eight) TRIM points in the bulk Brillouin zone. On the other hand, determining the $Z_{2}$ invariants for systems without space inversion symmetry is more compli- cated. While the $Z_{2}$ invariants can be expressed in terms of skew-symmetric matrices comprising the matrix elements of time-reversal operator between valence-band wave functions at the TRIM points $[16,17,19,20]$, numerical evaluation of the formula is not straightforward, since the phase of the valence-band wave functions must be chosen continuously in the Brillouin zone. A few alternative methods have been developed so far to overcome the difficulty. One of them is to use an expression for the $Z_{2}$ invariant that involves the line integral of the Berry connection and the surface integral of the Berry curvature of valence-band wave functions over half the 2D Brillouin zone [17]. Fukui and Hatsugai developed an efficient numerical algorithm to evaluate these integrals [22]. Another approach for determining the $Z_{2}$ invariant utilizes the Wannier function [23]. In this approach, one calculates the change in time-reversal polarization between two TRIM points from the evolution of the Wannier function centers of the occupied bands [24-26]. The expression derived by $\mathrm{Yu}$ et al. uses the non-Abelian Berry connection and is free from the gauge-fixing problem of valence band wave functions [25].

In the present work, we will demonstrate that the embedding potential as invented by Inglesfield [27,28] can be used to determine the topological invariants of time-reversal invariant band insulators. Given a semi-infinite crystal composed of atomic planes stacked in the surface normal direction and its boundary surface $S$, the embedding potential relates the value and normal derivative on $S$ of generalized Bloch states with energy $\epsilon$ and 2D wave vector $\mathbf{k}$, which propagate or decay toward the interior of the crystal. Essentially, it is a bulk quantity, as are the generalized Bloch states. The embedding method has been successfully used for first-principles calculations of the electronic structure of semi-infinite surfaces and interfaces [29-35].

As will be shown, the embedding potential becomes a Hermitian matrix having real eigenvalues if $\epsilon$ is located in a projected bulk band gap at a given $\mathbf{k}$. If one draws a line 
graph of these eigenvalues along a path in the SBZ, $\epsilon=\epsilon_{0}(\mathbf{k})$, passing between two TRIM points, $\mathbf{k}_{\alpha}$ and $\mathbf{k}_{\beta}$, these lines form Kramers doublets at both end points. There are two distinct ways these lines are connected between the two end points. In one case, two states forming a Kramers pair at $\mathbf{k}_{\alpha}$ meet again at $\mathbf{k}_{\beta}$ to form a Kramers pair. In the other case, the two states forming a Kramers pair at $\mathbf{k}_{\alpha}$ change partners at $\mathbf{k}_{\beta}$. These two cases remind us of the two distinct ways the surface bands in a projected bulk band gap disperse with $\mathbf{k}$ along a path connecting two TRIM points [19,20]. In fact, we will show that the former corresponds to the topologically trivial case with the associated $Z_{2}$ invariant $v=0$ and the latter corresponds to the topologically nontrivial case with $v=1$. Furthermore, we will show that these two cases are clearly classified by the number of poles, $P$, that the embedding potential exhibits along the path: the number of poles is even when $v=0$ and odd when $v=1$. In other words, we have $v=P \bmod 2$. The present formalism may be useful for calculating the $Z_{2}$ topological invariants of band insulators without space inversion centers, since the numerical procedure for calculating the embedding potential does not depend on whether the system has space inversion symmetry or not.

The plan of the present paper is as follows. In Sec. II, we present the main theory: starting with the definition of the embedding potential, we explain how it can be constructed from generalized Bloch states. Further, we show that its eigenvalues are real in a projected bulk band gap and discuss the properties of the eigenvalues when the system has timereversal symmetry. Then, we will show that the two distinct ways the eigenvalues of the embedding potential behave along a path linking two TRIM points is related to the associated $Z_{2}$ invariant. As an application of the theory, we study in Sec. III the embedding-potential eigenvalues of realistic materials by a first-principles calculation within density-functional theory (DFT). We choose CdTe, GeTe, and Bi as trivial insulators and $\mathrm{TlBiSe}_{2}$ and $\mathrm{Bi}_{2} \mathrm{Se}_{3}$ as topological insulators. Section IV contains some more theoretical discussions. We will clarify under what conditions surface bands crossing the bulk band gap inevitably emerge from a view point of embedding theory. We conclude in Sec. V. Unless otherwise stated, we use the Hartree atomic units throughout the present paper.

\section{THEORY}

\section{A. Definition of embedding potential}

We consider a bulk crystalline insulator composed of atomic lattice planes stacked in the $z$ direction. Two fundamental lattice vectors within the $x y$ plane are denoted by $\left\{\mathbf{a}_{1}, \mathbf{a}_{2}\right\}$, and the third one connecting two lattice points on two equivalent lattice planes with the shortest distance in the $z$ direction is denoted by $\mathbf{d}=\left(\mathbf{d}_{\|}, d_{z}\right)$ with $d_{z}$ chosen as a positive. The three-dimensional (3D) unit cell, i.e., the parallelepiped made out of $\left\{\mathbf{a}_{1}, \mathbf{a}_{2}, \mathbf{d}\right\}$ may contain more than one atomic planes. For example, $\mathrm{TlBiSe}_{2}$ and $\mathrm{Bi}_{2} \mathrm{Se}_{3}$ contain four and five atomic layers in a single unit cell when the $z$ axis is chosen as the [111] crystal orientation.

We divide the crystal into the left half-space and the right one by introducing a surface $S$, where it is assumed that the $z$ axis points to the right. In the simplest case, $S$ may be a cut-plane $z=z_{0}$. Let us consider $\psi$, an arbitrary solution of the Schrödinger equation with energy $\epsilon$ and 2D wave vector in the $x y$ plane, $\mathbf{k}$, satisfying the outgoing boundary condition at $z=-\infty$. In other words, $\psi$ decays or propagates toward the interior of the left half-space. The embedding potential for the left half-space relates the value and normal derivative of $\psi$ on $S$ by

$$
\partial_{n} \psi(\xi)=2 \int_{S} d \xi^{\prime} \Sigma_{L}\left(\mathbf{k}, \epsilon, \xi, \xi^{\prime}\right) \psi\left(\xi^{\prime}\right),
$$

where the surface normal $n$ points inward to the left half-space, and the factor 2 on the right-hand side is used to be consistent with the original definition of the embedding potential by Inglesfield [27], and we introduced a composite index $\xi=$ $(\mathbf{x}, \sigma)$ comprising a $2 \mathrm{D}$ space coordinate on $S, \mathbf{x}$, and spin index $\sigma$. Also, symbol $d \xi$ is understood to mean not only the surface integral over $S$ but also a summation over the spin index.

One may rewrite Eq. (1) in a discrete matrix form by introducing an orthonormal basis set defined on $S,\left\{\chi_{j}(\mathbf{x})\right\}$ $(j=1,2, \ldots)$ as

$$
\left\langle\eta \mid \partial_{n} \psi\right\rangle=2 \sum_{\eta^{\prime}}\left\langle\eta\left|\hat{\Sigma}_{L}(\mathbf{k}, \epsilon)\right| \eta^{\prime}\right\rangle\left\langle\eta^{\prime} \mid \psi\right\rangle,
$$

where $\eta=(j, \sigma)$ is a composite index specifying both basis function $\chi_{j}(\mathbf{x})$ and spin index $\sigma$. Here, $\langle\eta \mid \psi\rangle$ is defined by

$$
\langle\eta \mid \psi\rangle=\int_{S} d \mathbf{x} \chi_{j}^{*}(\mathbf{x}) \psi(\mathbf{x}, \sigma),
$$

and $\left\langle\eta \mid \partial_{n} \psi\right\rangle$ and $\left\langle\eta\left|\hat{\Sigma}_{L}(\mathbf{k}, \epsilon)\right| \eta^{\prime}\right\rangle$ are defined in the same way. Hereafter, we use " $\wedge$ " to explicitly indicate that the quantity to which this symbol is attached is an operator or matrix. In the following, a discrete representation using the basis set $\{|\eta\rangle\}$ is called " $\eta$ representation." For example, if $S$ is a planar surface $z=z_{0}$, one may employ plane-wave basis functions,

$$
\chi_{j}(\mathbf{x})=\frac{1}{\sqrt{A}} \exp \left[i\left(\mathbf{k}+\mathbf{g}_{j}\right) \cdot \mathbf{x}\right],
$$

where $\mathbf{g}_{j}$ denotes $2 \mathrm{D}$ reciprocal lattice vectors in the $x y$ plane and $A$ is the normalization area of $S$. In actual calculations, the number of basis functions can be chosen to be finite by introducing some cutoff parameter. For example, for the plane-wave basis set, Eq. (4), one may introduce a cutoff energy $E_{\text {cut }}=G_{\text {cut }}^{2} / 2$ and include only plane waves satisfying the condition $\left|\mathbf{k}+\mathbf{g}_{j}\right| \leqslant G_{\text {cut }}$ in the basis set. In this case, the embedding potential in $\eta$ representation becomes a $2 N \times 2 N$ matrix including the spin degree of freedom, where $N$ is the number of the reciprocal lattice vectors included in the basis set.

\section{B. How to construct embedding potential}

To construct the embedding potential, one first calculates the wave functions with energy $\epsilon$ and $2 \mathrm{D}$ wave vector $\mathbf{k}$ satisfying the generalized Bloch condition,

$$
\begin{aligned}
\psi(\mathbf{x}+\mathbf{d}, \sigma) & =\mu \psi(\mathbf{x}, \sigma), \\
\partial_{n} \psi(\mathbf{x}+\mathbf{d}, \sigma) & =\mu \partial_{n} \psi(\mathbf{x}, \sigma),
\end{aligned}
$$

with

$$
\mu=\exp \left(i \mathbf{k} \cdot \mathbf{d}_{\|}+i q_{z} d_{z}\right),
$$


as the eigenvectors of the transfer matrix relating the value and normal derivative of wave functions on $S$ with the corresponding ones on the neighboring surface shifted by $\mathbf{d}$. A method to construct such a transfer matrix based on Green's theorem was given in Ref. [32]. In Eq. (6), $q_{z}$ is a complex wave number. Bloch waves correspond to $\psi$ 's with $\operatorname{Im} q_{z}=0$, while $\psi$ 's with positive (negative) $\operatorname{Im} q_{z}$ are evanescent waves that decay toward $z=+\infty(-\infty)$. In $\eta$ representation, the transfer matrix becomes a $4 N \times 4 N$ matrix (including spin degree of freedom). Among its $4 N$ eigenvalues, half of them, i.e., $2 N$ solutions correspond to $\psi$ 's propagating or decaying toward $z=-\infty$. Let us denote the value and normal derivative of these $2 N$ solutions on $S$ by $\psi_{j}$ and $\partial_{n} \psi_{j}$. Then, by using Eq. (2), the $2 N \times 2 N$ embedding-potential matrix for the left half-space can be expressed as

$$
\hat{\Sigma}_{L}(\mathbf{k}, \epsilon)=\frac{1}{2} \partial_{n} \hat{\Psi} \hat{\Psi}^{-1},
$$

where $(\hat{\Psi})_{\eta j}=\left\langle\eta \mid \psi_{j}\right\rangle$ and $\left(\partial_{n} \hat{\Psi}\right)_{\eta j}=\left\langle\eta \mid \partial_{n} \psi_{j}\right\rangle$ with $\eta$ and $j$ ranging from 1 to $2 N$.

It should be emphasized that one needs no semi-infinite calculations to compute $\hat{\Sigma}_{L}(\mathbf{k}, \epsilon)$. The generalized Bloch states in Eq. (7) are obtained by a calculation involving only one bulk unit cell.

\section{Eigenvalue spectra of embedding potential}

As before, we denote an arbitrary wave function with energy $\epsilon$ and 2D wave vector $\mathbf{k}$ propagating or decaying toward $z=$ $-\infty$ by $\psi$. By using Eq. (1), the current through $S$ carried by $\psi$ is given by

$$
\begin{aligned}
J & =\frac{1}{2} \int_{S}\left[\psi^{*}(\xi) \partial_{n} \psi(\xi)-\partial_{n} \psi^{*}(\xi) \psi(\xi)\right] d \xi \\
& =\frac{1}{2} \int_{S} \psi^{*}(\xi)\left[\Sigma_{L}\left(\xi, \xi^{\prime}\right)-\Sigma_{L}^{*}\left(\xi^{\prime}, \xi\right)\right] \psi\left(\xi^{\prime}\right) d \xi d \xi^{\prime},
\end{aligned}
$$

where we omitted two arguments, $\epsilon$ and $\mathbf{k}$, for simplicity. If $\epsilon$ is located in a projected bulk band at a given $\mathbf{k}$, i.e., if there are Bloch waves with a $3 \mathrm{D}$ wave vector $\left(\mathbf{k}, \operatorname{Re} q_{z}\right)$ and energy $\epsilon$ propagating toward $z=-\infty, J$ may become positive. On the other hand, if $\epsilon$ is located in a projected bulk band gap, i.e., if all the solutions of Eq. (5) are evanescent waves with nonzero $\operatorname{Im} q_{z}$, then, $J$ must vanish. This means that within a projected bulk band gap, the embedding potential is Hermitian, i.e.,

$$
\Sigma_{L}\left(\mathbf{k}, \epsilon, \xi, \xi^{\prime}\right)=\Sigma_{L}^{*}\left(\mathbf{k}, \epsilon, \xi^{\prime}, \xi\right) .
$$

This signifies that all the eigenvalues of the embedding potential are real. Let us denote the $i$-th eigenvalue of the embedding potential $\hat{\Sigma}(\mathbf{k}, \epsilon)$ by $\lambda_{i}(\mathbf{k}, \epsilon)$ and the corresponding eigenfunction by $\phi_{i}$, namely, in $\eta$ representation,

$$
\sum_{\eta^{\prime}}\left\langle\eta\left|\hat{\Sigma}_{L}(\mathbf{k}, \epsilon)\right| \eta^{\prime}\right\rangle\left\langle\eta^{\prime} \mid \phi_{i}\right\rangle=\lambda_{i}(\mathbf{k}, \epsilon)\left\langle\eta \mid \phi_{i}\right\rangle .
$$

By definition of the embedding potential (1), this implies that

$$
\partial_{n} \phi_{i}=2 \lambda_{i}(\mathbf{k}, \epsilon) \phi_{i},
$$

holds on $S$. That is, by appropriately taking a linear combination of the evanescent waves with a negative $\operatorname{Im} q_{z}$ satisfying Eq. (5), one can construct an evanescent wave $\phi_{i}$, whose logarithmic normal derivative takes a constant value $2 \lambda_{i}$ on every point on $S$ for both spin components. We normalize $\phi_{i}$ on $S$. Then, since eigenfunctions of a Hermitian matrix are orthogonal to one another, we have

$$
\hat{\Sigma}_{L}(\mathbf{k}, \epsilon)=\sum_{i} \lambda_{i}(\mathbf{k}, \epsilon)\left|\phi_{i}\right\rangle\left\langle\phi_{i}\right| .
$$

Also, the determinant of $\hat{\Sigma}_{L}(\mathbf{k}, \epsilon)$ is given by

$$
\operatorname{det}\left[\hat{\Sigma}_{L}(\mathbf{k}, \epsilon)\right]=\prod_{i} \lambda_{i}(\mathbf{k}, \epsilon)
$$

An expression similar to Eq. (12) was derived for the imaginary part of the embedding potential previously by Inglesfield et al. [36] in the discussion of current-carrying channel functions. In contrast, we consider in the present work energies in a projected bulk band gap where no Bloch states exist.

\section{Time-reversal symmetry}

Now, we assume that the band insulator under consideration is invariant with respect to time-reversal operation $\hat{T}=$ $-i \hat{\sigma}_{y} \hat{K}$, where $\hat{\sigma}_{y}$ is the $y$ component of Pauli matrices and $\hat{K}$ is a complex-conjugate operator. By operating $\hat{T}$ on both sides of Eq. (11) and noting that $\lambda_{i}$ is real, one obtains

$$
\partial_{n}\left(\hat{T} \phi_{i}\right)=2 \lambda_{i}(\mathbf{k}, \epsilon)\left(\hat{T} \phi_{i}\right) .
$$

$\hat{T} \phi_{i}$ is an evanescent wave decaying toward $z=-\infty$ with energy $\epsilon$ and a 2D wave vector $-\mathbf{k}$. Namely, $\hat{T} \phi_{i}$ is an eigenfunction of the embedding potential $\hat{\Sigma}_{L}(-\mathbf{k}, \epsilon)$. As a result,

$$
\lambda_{i}(-\mathbf{k}, \epsilon)=\lambda_{i}(\mathbf{k}, \epsilon) .
$$

Also, it is easy to show that

$$
\hat{\Sigma}_{L}(-\mathbf{k}, \epsilon)=\hat{T} \hat{\Sigma}_{L}(\mathbf{k}, \epsilon) \hat{T}^{-1},
$$

where $\hat{T}^{-1}=-\hat{T}$. It should be emphasized that the above equation holds only when the energy $\epsilon$ is located in a projected bulk band gap for a given $\mathbf{k}$.

Let us consider the four TRIM points in the SBZ, i.e., $\mathbf{k}_{\mathrm{I}}=\mathbf{0}, \quad \mathbf{k}_{\mathrm{II}}=\mathbf{b}_{1} / 2, \quad \mathbf{k}_{\mathrm{III}}=\mathbf{b}_{2} / 2, \quad$ and $\quad \mathbf{k}_{\mathrm{IV}}=\left(\mathbf{b}_{1}+\mathbf{b}_{2}\right) / 2$, where $\mathbf{b}_{1}$ and $\mathbf{b}_{2}$ are 2D fundamental reciprocal lattice vectors satisfying the conditions

$$
\mathbf{a}_{i} \cdot \mathbf{b}_{j}=2 \pi \delta_{i j}
$$

At these $\mathbf{k}$ points, $\mathbf{k}$ and $-\mathbf{k}$ are equivalent because they are connected by a reciprocal lattice vector. Thus one sees from the above equations that the eigenvalues of the embedding potential are twofold degenerate, forming Kramers pairs at $\mathbf{k}_{\alpha}$ $(\alpha=\mathrm{I} \sim \mathrm{IV})$.

In the 3D Brillouin zone, there are eight time-reversal invariant $3 \mathrm{D}$ wave vectors, whose projection onto the $k_{x} k_{y}$ plane coincides with one of the four 2D TRIM points. That is, each $\mathbf{k}_{\alpha}(\alpha=\mathrm{I} \sim \mathrm{IV})$ is associated with two timereversal invariant 3D wave vectors, $\mathbf{K}_{\alpha}^{i}(i=1,2)$, whose planar component is $\mathbf{k}_{\alpha}$, while the $k_{z}$ components of $\mathbf{K}_{\alpha}^{1}$ and $\mathbf{K}_{\alpha}^{2}$ differ by $\pi / d_{z}[19,20]$. 
The time-reversal polarization associated with $\mathbf{k}_{\alpha}$ is defined by $\pi_{\alpha}=\delta_{\alpha}^{1} \delta_{\alpha}^{2}$ where $\delta_{\alpha}^{i}$ is [17]

$$
\delta_{\alpha}^{i}=\frac{\sqrt{\operatorname{det}\left[\hat{w}\left(\mathbf{K}_{\alpha}^{i}\right)\right]}}{\operatorname{Pf}\left[\hat{w}\left(\mathbf{K}_{\alpha}^{i}\right)\right]},
$$

where $\hat{w}$ is a $2 M \times 2 M$ skew-symmetric matrix constructed from the valence-band wave functions, with $2 M$ being the number of valence bands including the spin degree of freedom.

In the following discussion, the $2 \mathrm{D}$ wave vector $\mathbf{k}$ is assumed to be on a path connecting two TRIM points, $\mathbf{k}_{\alpha}$ and $\mathbf{k}_{\beta}$. For simplicity, we use a scalar variable $k$ to represent $\mathbf{k}$ on the path in the SBZ. In Fig. 1, we show a schematic view of 2D surface $\lambda=\lambda_{i}(k, \epsilon)$ in the 3D Cartesian coordinate system with the $k, \epsilon$, and $\lambda$ axis lines, where the $k \epsilon$ plane is chosen as the horizontal plane and the $\lambda$ axis points to the vertical direction. It should be noted that this surface is defined only inside the projected band gap $\epsilon_{v}(k)<\epsilon<\epsilon_{c}(k)$. The shaded region $\epsilon \geqslant \epsilon_{c}(k)\left(\epsilon \leqslant \epsilon_{v}(k)\right)$ corresponds to the energy continuum of bulk conduction (valence) bands.

Let us consider the lines of intersection between surfaces $\lambda=\lambda_{i}(k, \epsilon)(i=1,2, \ldots)$ and a vertical cut-plane $\epsilon=\epsilon_{0}$ with $\epsilon_{0}$ located in the projected bulk band gap. As discussed above, at both $k_{\alpha}$ and $k_{\beta}$, these lines form Kramers doublets. In Fig. 1(b), we show two possible ways how these lines are connected between $k_{\alpha}$ and $k_{\beta}$. In the left panel, two states in a Kramers pair at $k_{\alpha}$ meet again at $k_{\beta}$ to form a Kramers pair. As a result, one would have couples of lines which are connected both at $k_{\alpha}$ and $k_{\beta}$, and are separated from the neighboring pairs of lines. In this case, there may be a " $\lambda$ gap" between two pairs of lines, i.e., a range of $\lambda$ values in which no $\lambda=\lambda_{i}\left(k, \epsilon_{0}\right)$ lines enter. On the other hand, in the right panel, each state in a Kramers pair at $k_{\alpha}$ changes the partner at $k_{\beta}$ to meet a state that belongs to a neighboring Kramers pair at $k_{\alpha}$. In this case, the set of line segments $\lambda=\lambda_{i}\left(k, \epsilon_{0}\right)(i=1,2, \ldots)$ may be regarded as a single continuous line alternating between $k_{\alpha}$ and $k_{\beta}$ and ranging between $\lambda=-\infty$ and $+\infty$. It is important that the uppermost line $\lambda=\lambda_{1}\left(k, \epsilon_{0}\right)$ should inevitably diverge to $+\infty$ at $k_{0}$ between $k_{\alpha}$ and $k_{\beta}$. Correspondingly, the lowermost line on the negative side of $\lambda$ diverges to $-\infty$ at $k_{0}$. In other words, if one plots $1 / \lambda_{i}$ as a function of $k$, the first and last branches are smoothly connected at $k=k_{0}$. As seen from Eq. (13), $k_{0}$ corresponds to a pole of the determinant $\operatorname{det}\left[\hat{\Sigma}_{L}\left(k, \epsilon_{0}\right)\right]$. Furthermore, as seen from Eq. (7), the pole corresponds to a zero of $\operatorname{det}[\hat{\Psi}]$. Physically, this means that one can construct a solution of the Schrödinger equation with energy $\epsilon_{0}$ and wave number $k_{0}$ whose amplitude on $S$ vanishes identically from a linear combination of the $2 N$ evanescent waves satisfying the generalized Bloch condition Eq, (5). In contrast to the left panel, there appears no " $\lambda$ gap," and any $\lambda$ value between $-\infty$ and $+\infty$ becomes an eigenvalue of $\hat{\Sigma}_{L}\left(k, \epsilon_{0}\right)$ at some $k$ between $k_{\alpha}$ and $k_{\beta}$.

The above discussion on the two possible ways how the eigenvalues of the embedding potential behave with $k$ may remind us of the two possible ways how the energy dispersion relations with $k$ of the surface states on a semi-infinite timereversal invariant insulator behave in a projected bulk band gap $[19,20]$. As shown in Fig. 1(c), due to time-reversal symmetry, the surface states are doubly degenerate at the two TRIM points, $k_{\alpha}$ and $k_{\beta}$. As is known $[19,20]$, if $(-1)^{v}=\pi_{\alpha} \pi_{\beta}=1$

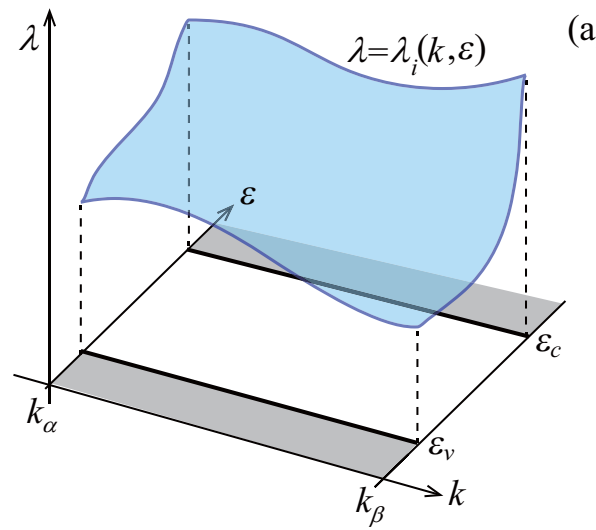

(a)

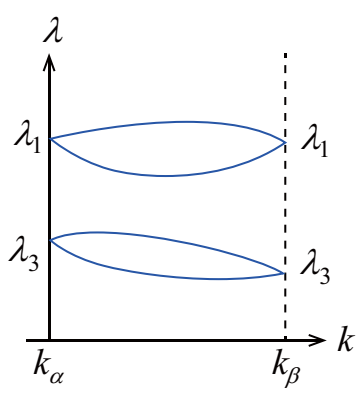

(b)
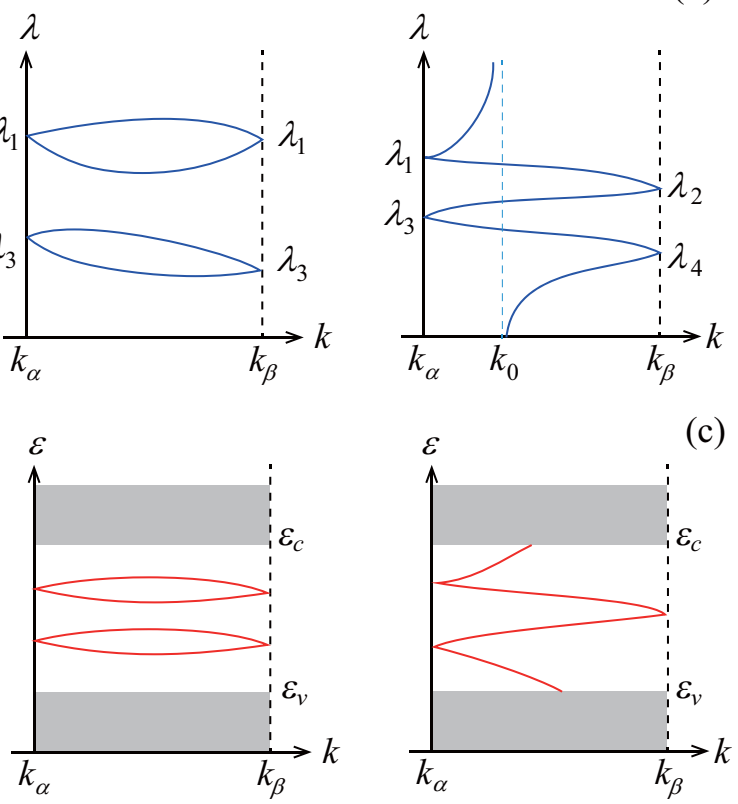

FIG. 1. (a) Schematic view of the $2 \mathrm{D}$ surface $\lambda=\lambda_{i}(k, \epsilon)$ in a 3D $(k, \epsilon, \lambda)$ coordinate space. The surface is defined only inside the projected bulk band gap $\epsilon_{v}(k)<\epsilon<\epsilon_{c}(k)$. The upper (lower) shaded area on the $k \epsilon$ plane corresponds to the energy continuum of bulk conduction (valence) bands. $k_{a}$ and $k_{b}$ denote two TRIM points in the SBZ. (b) Lines of intersection between surfaces $\lambda=\lambda_{i}(k, \epsilon)(i=$ $1,2, \ldots)$ and a vertical cut plane $\epsilon=\epsilon_{0}$ plotted as a function of $k$ where $\epsilon_{0}$ is located inside the projected band gap. In the left panel, two states forming a Kramers pair at $k_{\alpha}$ are connected again at $k_{\beta}$. In the right panel, two states forming a Kramers pair at $k_{\alpha}$ change their partners at $k_{\beta}$. (c) Energy dispersion relation with 2D wave number $k$ of the surface states of semi-infinite band insulators inside the projected bulk band gap along a path connecting two TRIM points. Left and right panels correspond to $\pi_{\alpha} \pi_{\beta}=1$ and -1 , where $\pi_{\alpha}\left(\pi_{\beta}\right)$ denotes time-reversal polarization at $k_{\alpha}\left(k_{\beta}\right)$.

$(v=0)$, two surface states in a Kramers pair at $k_{\alpha}$ meet each other again at $k_{\beta}$ (see the left panel). On the other hand, if $\pi_{\alpha} \pi_{\beta}=-1(\nu=1)$, each surface state in a Kramers pair at $k_{\alpha}$ changes the partner to become degenerate at $k_{\beta}$ with another surface state which belongs to a neighboring Kramers pair at $k_{\alpha}$ (see the right panel). It may be tempting from the close similarity between Figs. 1(b) and 1(c) to infer that the left panel in Fig. 1(b) may correspond to the topologically trivial case with $\pi_{\alpha} \pi_{\beta}=1$, whereas the right panel may correspond 
to the topologically nontrivial case with $\pi_{\alpha} \pi_{\beta}=-1$. In the next section. we will show that this is actually the case.

\section{E. Surface states}

In Sec. II A, we introduced surface $S$ to divide a 3D band insulator into the left and right half-spaces. Now, we consider a semi-infinite crystal to the left of $S$. The surface states appearing in the projected bulk band gap between its bulk valence and conduction bands are determined by the boundary condition of electron wave functions on $S$. In Sec. IV, we will consider realistic boundary conditions taking account of surface atomic layers and the semi-infinite vacuum. However, here we consider a more simplified mathematical boundary condition on $S$, i.e.,

$$
\partial_{n} \phi(\xi)=2 \lambda_{0} \phi(\xi),
$$

with a constant $\lambda_{0}$. For example, $\lambda_{0}=0$ corresponds to the Neumann boundary condition with $\partial_{n} \phi(\xi)=0$ on $S$, while $\lambda_{0}= \pm \infty$ corresponds to the Dirichlet boundary condition with $\phi(\xi)=0$ on $S$. Surface states inside the projected bulk band gap are the evanescent waves satisfying Eq. (18). On the other hand, this equation has exactly the same form as that for the eigenfunctions of the embedding potential (11). Accordingly, the energy dispersion relations of the surface states with $k$ are given by the lines of intersection between the horizontal plane $\lambda=\lambda_{0}$ and the eigenvalue surfaces of the embedding potential, $\lambda=\lambda_{i}(k, \epsilon)(i=1,2, \ldots)$, shown in Fig. 1(a). This may give us a clear relationship between Figs. 1(b) and 1(c): namely, the eigenvalue dispersion curves with $k$ shown in Fig. 1(b) are the lines of intersection between 2D surfaces $\lambda=\lambda_{i}(k, \epsilon)(i=1,2, \ldots)$ and a vertical cut plane $\epsilon=\epsilon_{0}$, whereas the surface-state energy dispersion relations with $k$ shown in Fig. 1(c) are the lines of intersection between the same $2 \mathrm{D}$ surfaces and a horizontal cut plane $\lambda=\lambda_{0}$. How these eigenvalue surfaces intersect with a horizontal or a vertical cut plane may be determined by the geometrical behaviors of these 2D surfaces themselves.

As was shown previously $[19,20]$, regardless of the boundary conditions at the surface, there appear in the projected bulk band gap, surface bands whose energy dispersion relation with $k$ behaves like the right panel of Fig. 1(c) if $\pi_{\alpha} \pi_{\beta}=-1$ $(v=1)$. This indicates that, no matter what the value of $\lambda_{0}$ is, the lines of intersection between 2D surfaces $\lambda=\lambda_{i}(k, \epsilon)$ $(i=1,2, \ldots)$ and the cut plane $\lambda=\lambda_{0}$ look like the right panel of Fig. 1(c) if $v=1$. One may reconstruct the original 2D surfaces $\lambda=\lambda_{i}(k, \epsilon)(i=1,2, \ldots)$ from these lines of intersection by continuously varying the value of $\lambda_{0}$ from $-\infty$ to $+\infty$. Then, obviously, the lines of intersection between the surfaces $\lambda=\lambda_{i}(k, \epsilon)(i=1,2, \ldots)$ reconstructed in this manner and a vertical cut-plane $\epsilon=\epsilon_{0}$ should be of the type as given in the right panel of Fig. 1(b). The same argument holds for the other case with $\pi_{\alpha} \pi_{\beta}=1(\nu=0)$. In this case, the lines of intersection between 2D surfaces $\lambda=\lambda_{i}(k, \epsilon)$ $(i=1,2, \ldots)$ and a cut-plane $\lambda=\lambda_{0}$ behave like the left panel of Fig. 1(c) for any value of $\lambda_{0}$, unless $\lambda_{0}$ is located within a " $\lambda$ gap" where no surface states occur. Then, the lines of intersection between the same surfaces and a cut plane $\epsilon=\epsilon_{0}$ should behave like the left panel of Fig. 1(b). To summarize, the embedding potential within the projected bulk band gap between the valence and conduction bands should exhibit qualitatively different eigenvalue spectra along a path connecting two TRIM points, $k_{\alpha}$ and $k_{\beta}$, depending on the value of the topological invariant of the valence band structure.

\section{APPLICATION}

\section{A. Calculational method}

To demonstrate that the theoretical results derived in Sec. II hold true, we calculate the embedding potential and its eigenvalues of several band insulators by a first-principles method within DFT. For this purpose, we use a computer code $[34,35]$ that combines the full-potential linearized augmented plane-wave (LAPW) method [37] and the embedded Green's function technique of Inglesfield [27,28]. The exchangecorrelation energy is treated within the generalized gradient approximation by using the energy functional of Perdew, Burke, and Ernzerhof [38].

For a given crystal orientation, we consider a slab-shaped region with the left boundary surface $S$ and the right one $S^{\prime}$. The slab contains a single unit cell in the surface normal direction, and $S$ and $S^{\prime}$ are related by the lattice vector d defined in Sec. II A. In most cases, any planar surface between two neighboring atomic layers intersects the muffin-tin (MT) spheres of atoms on both sides of the plane. This is not convenient from a numerical point of view, since we use the LAPW basis functions. To avoid this, when we construct the boundary surface $S$, we start with a planar surface $z=z_{0}$ at the middle of two neighboring atomic planes, and if the plane $z=z_{0}$ cuts the MT sphere of a nearby atom whose nucleus is located on the right (left) side of $z=z_{0}$, we remove the disk-shaped small portion of $z=z_{0}$ contained inside the MT sphere of this atom from $S$, and instead add to $S$ the cap-shaped small portion of the MT spherical surface to the left (or right) of the plane $z=z_{0}$, so that the MT sphere of this atom may be located entirely to the right (or left) of the resultant curvy surface. This procedure is repeated for all the atoms whose MT spheres intersect $z=z_{0}$. As basis functions to expand the wave function defined on the resultant curvy surface $S$, we employ [39]

$$
\chi_{j}(\mathbf{x})=\frac{1}{\sqrt{A}} \exp \left[i\left(\mathbf{k}+\mathbf{g}_{j}\right) \cdot \tilde{\mathbf{x}}\right],
$$

where $\mathbf{g}_{j}$ denotes a 2D reciprocal lattice vector in the $x y$ plane as before, and $\mathbf{x}=(\tilde{\mathbf{x}}, z(\tilde{\mathbf{x}}))$ is a point on $S$ with $\tilde{\mathbf{x}}=(x, y)$ being its projection onto the $x y$ plane. As far as $\left(\tilde{\mathbf{x}}, z_{0}\right)$ is located in the interstitial region, $z(\tilde{\mathbf{x}})$ coincides with $z_{0}$. Only when $\left(\tilde{\mathbf{x}}, z_{0}\right)$ is located inside the MT sphere of a nearby atom, $z(\tilde{\mathbf{x}})$ deviates from $z_{0}$ so that $(\tilde{\mathbf{x}}, z(\tilde{\mathbf{x}}))$ may be on the cap-shaped surface of the MT sphere of the atom.

Since the basis set $\{|\eta\rangle\}$ with $\eta=(j, \sigma)(\eta=1,2, \ldots, 2 N)$, where $N$ is the number of the reciprocal lattice vectors included in the basis set, is nonorthogonal on $S$, the equations in " $\eta$ representation" given in Sec. II must be slightly modified. This is most easily handled by introducing the dual basis set $\{|\bar{\eta}\rangle\}(\eta=1,2, \ldots, 2 N)$, where $|\bar{\eta}\rangle$ is defined by

$$
|\bar{\eta}\rangle=\sum_{\eta^{\prime}}\left|\eta^{\prime}\right\rangle\left(S^{-1}\right)_{\eta^{\prime} \eta},
$$


with $S_{\eta \eta^{\prime}}=\left\langle\eta \mid \eta^{\prime}\right\rangle$. The dual basis set satisfies $\left\langle\bar{\eta} \mid \eta^{\prime}\right\rangle=\delta_{\eta \eta^{\prime}}$. With these notations, Eq. (2) should now read

$$
\left\langle\bar{\eta} \mid \partial_{n} \psi\right\rangle=2 \sum_{\eta^{\prime}}\left\langle\bar{\eta}\left|\hat{\Sigma}_{L}(\mathbf{k}, \epsilon)\right| \bar{\eta}^{\prime}\right\rangle\left\langle\eta^{\prime} \mid \psi\right\rangle .
$$

Similarly, the eigenvalue equation of the embedding potential Eq. (10) is modified as

$$
\sum_{\eta^{\prime}}\left\langle\bar{\eta}\left|\hat{\Sigma}_{L}(\mathbf{k}, \epsilon)\right| \bar{\eta}^{\prime}\right\rangle\left\langle\eta^{\prime} \mid \phi_{i}\right\rangle=\lambda_{i}(\mathbf{k}, \epsilon) \sum_{\eta^{\prime}}\left\langle\bar{\eta} \mid \bar{\eta}^{\prime}\right\rangle\left\langle\eta^{\prime} \mid \phi_{i}\right\rangle,
$$

where $\left\langle\bar{\eta} \mid \bar{\eta}^{\prime}\right\rangle=\left(S^{-1}\right)_{\eta \eta^{\prime}}$.

As mentioned in Sec. II B, the embedding potential is constructed from the transfer matrix that relates the value and normal derivative of wave functions on $S$ and $S^{\prime}$. More details on the computational method of the transfer matrix and the generalized Bloch states are given in Ref. [32].

\section{B. Empty lattice}

Before presenting results for realistic materials, we discuss some general aspects of the eigenvalues of the embedding potential by adopting the empty lattice approximation. We assume that the potential energy takes a constant value $E_{0}$ in the whole space and choose the plane $z=z_{0}$ as the embedding surface. Then, the solution of the Schrödinger equation with energy $\epsilon$ and $2 \mathrm{D}$ wave vector $\mathbf{k}$ satisfying the outgoing boundary condition at $z=-\infty$ is a plane wave or an evanescent wave of the form containing a single $2 \mathrm{D}$ reciprocal lattice vector $\mathbf{k}+\mathbf{g}_{j}$,

$$
\psi(\mathbf{r})=\frac{1}{\sqrt{S}} \exp \left[i\left(\mathbf{k}+\mathbf{g}_{j}\right) \cdot \mathbf{x}+\kappa_{z} z\right]
$$

Thus $\hat{\Sigma}_{L}(\mathbf{k}, \epsilon)$, the embedding potential for the left half-space, is diagonal with respect to $2 \mathrm{D}$ reciprocal lattice vectors and its eigenvalues are given by

$$
\lambda_{j}=-\frac{1}{2} \kappa_{z}=-\frac{1}{2} \sqrt{\left|\mathbf{k}+\mathbf{g}_{j}\right|^{2}+2\left(E_{0}-\epsilon\right)},
$$

with $\operatorname{Im} \lambda_{i} \geqslant 0$. For the present case, the projected bulk band gap corresponds to $\epsilon<E_{0}+\frac{1}{2} \min _{i}\left|\mathbf{k}+\mathbf{g}_{i}\right|^{2}$, and all the eigenvalues $\lambda_{i}$ becomes real and negative in the projected bulk band gap.

As an example, let us consider a 2D hexagonal lattice with lattice constant $a$ as the lattice structure in the $x y$ plane. The real-space lattice vectors are $\mathbf{a}_{1}=(a, 0)$ and $\mathbf{a}_{2}=\left(\frac{1}{2} a, \frac{\sqrt{3}}{2} a\right)$, and the corresponding four TRIM points are $\mathbf{k}_{\mathrm{I}}=(0,0)(\Gamma)$, $\mathbf{k}_{\mathrm{II}}=\left(\frac{\pi}{a},-\frac{\pi}{\sqrt{3} a}\right), \mathbf{k}_{\mathrm{III}}=\left(0, \frac{2 \pi}{\sqrt{3} a}\right)$, and $\mathbf{k}_{\mathrm{IV}}=\left(\frac{\pi}{a}, \frac{\pi}{\sqrt{3} a}\right)$. In the following sections, we consider semi-infinite crystals having $\mathrm{C}_{3 v}$ symmetry, for which the latter three TRIM points are symmetrically equivalent (the $M$ point).

In Fig. 2(a), we show the embedding-potential eigenvalues of a semi-infinite crystal within the empty lattice approximation with a hexagonal-lattice structure in the $x y$ plane. Here, the lattice constant in the plane, electron energy, and cutoff parameter are chosen as $a=8.0$ a.u., $\epsilon=E_{0}-0.01$ a.u., and $G_{\text {cut }}=3.5$ a.u., respectively. Since $\lambda_{i}(k, \epsilon)$ as a function of $k$ can diverge at poles for realistic materials, in the present work, we have chosen to plot $\tan ^{-1} \lambda_{i}$ instead of $\lambda_{i}$ to show the embedding-potential eigenvalues. Thus the range
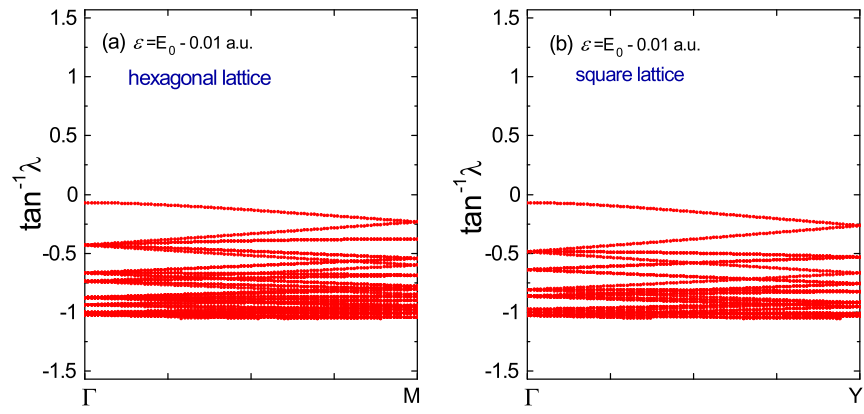

FIG. 2. Embedding-potential eigenvalues $\lambda_{i}(k, \epsilon)$ of semi-infinite crystals within the empty lattice approximation along a $\mathbf{k}$-space line segment connecting two TRIM points. (a) Hexagonal lattice with lattice constant $a=8$ a.u., (b) square lattice with $a=6$ a.u. In both panels, energy $\epsilon$ is $E_{0}-0.01$ a.u., and the cutoff parameter $G_{\text {cut }}$ is 3.5 a.u.

of the vertical axis becomes a finite interval $[-\pi / 2, \pi / 2]$. Each $\lambda=\lambda_{i}\left(k, \epsilon_{0}\right)$ line in Fig. 2(a) is doubly degenerate with respect to spin degrees of freedom, and more than one lines meet together at $\Gamma$ and $M$ because of the empty lattice approximation. The latter degeneracy can be lifted by introducing nonvanishing lattice potentials, while the former degeneracy can be lifted by introducing spin-orbit interactions except at the two TRIM points, $\Gamma$ and $M$. The $\lambda_{i}(k, \epsilon)$ values corresponding to eigenfunctions $\phi_{i}$ 's that decay slowly toward the interior of the crystal do not change upon increasing cutoff parameter $G_{\text {cut }}$, while one obtains additional eigenfunctions whose amplitude is strongly localized near $S$ by increasing $G_{\text {cut }}$. As will be shown in the following sections, these rapidly decaying solutions are rather insensitive to material details and form a bunch of $\lambda_{i}(k, \epsilon)$ bands whose lower limit is given by $\sim-\frac{1}{2} G_{\text {cut }}$. This is somewhat analogous to electronic band structure calculations of bulk crystals, in which the low-lying energy bands hardly change with increasing plane-wave cutoff parameter $G_{\text {cut }}$, while one obtains additional free-electron-like states with energy $\sim \frac{1}{2} G_{\text {cut }}^{2}$ by increasing $G_{\text {cut }}$.

For comparison, we show in Fig. 2(b) the $\lambda_{i}(k, \epsilon)$ values of a semi-infinite crystal within the empty lattice approximation with a square-lattice structure in the $x y$ plane, where the lattice constant $a=6.0$ a.u., $\epsilon=E_{0}-0.01$ a.u., $G_{\text {cut }}=3.5$ a.u., and the $Y$ point corresponds to $\mathbf{k}=\left(0, \frac{\pi}{a}\right)$. Qualitatively, the eigenvalue spectra in both panels are very similar. In the sense that both eigenvalue spectra exhibit no poles along the paths connecting two TRIM points, one may regard the half-space with constant potential $E_{0}$ as topologically trivial.

\section{C. $\operatorname{CdTe}(111)$}

Now, we present the calculated results for several realistic band insulators exhibiting strong spin-orbit interactions. All the systems considered in the present work possess the $\mathrm{C}_{3 v}$ symmetry and we choose the $y$ axis such that the $y z$ plane is one of the three mirror reflection planes. We begin with CdTe crystallized in the zinc-blende structure with a cubic lattice constant of $6.48 \AA$ [40]. This material is topologically trivial and characterized by $3 \mathrm{D} Z_{2}$ invariants, $\left(v_{0} ; v_{1} v_{2} v_{3}\right)=(0 ; 000)$. Since the bulk crystal has no space inversion symmetry, the standard parity analysis cannot be used to determine these $Z_{2}$ parameters. 
(a)
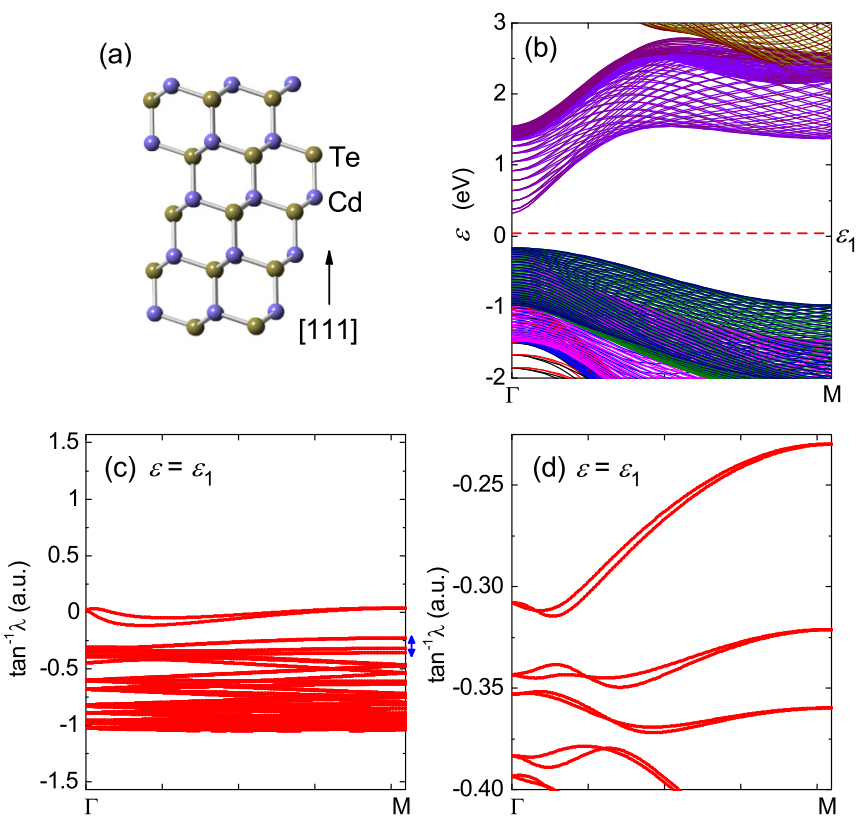

FIG. 3. (a) Crystal structure of CdTe seen from a direction perpendicular to the [111] axis. (b) Projected bulk bands of CdTe(111) along the $\Gamma-M$ line produced in two steps: first, one fixes the surface normal $(z)$ component of the wave vector, $k_{z}$, and plots the energy of each band with $2 \mathrm{D} \mathrm{k}$ (different bands are plotted in different colors). Second, one varies $k_{z}$ with a small mesh interval from $-\pi / d_{z}$ to $\pi / d_{z}$. (c) The eigenvalue spectrum of the embedding potential of $\mathrm{CdTe}(111)$ along a constant energy path $\epsilon=\epsilon_{1}$ shown by a dashed line in (b). (d) Enlargement of (c) corresponding to a small $\lambda$ interval indicated by the vertical blue arrow in (c).

We calculate the eigenvalues of $\hat{\Sigma}_{L}(k, \epsilon)$ for a semiinfinite $\operatorname{CdTe}(111)$ along a straight path between $\mathbf{k}_{\mathrm{I}}=(0,0)$ ( $\Gamma)$ and $\mathbf{k}_{\mathrm{III}}=\left(0, \frac{2 \pi}{\sqrt{3} a}\right)(M)$ with $a=6.48 / \sqrt{2} \AA$ being a hexagonal lattice constant of the (111) plane. Since the three TRIM points, $\mathbf{k}_{\mathrm{II}}, \mathbf{k}_{\mathrm{III}}$, and $\mathbf{k}_{\mathrm{IV}}$ are symmetrically equivalent, we have $\pi_{\Gamma} \pi_{M}=\pi_{\Gamma}\left(\pi_{M}\right)^{3}=\prod_{\alpha=\mathrm{I} \sim \mathrm{IV}} \pi_{\alpha}=(-1)^{\nu_{0}}=1$ (note that $(-1)^{\nu_{0}}$ equals the product of Eq. (17) for the eight 3D TRIM points). Thus it is expected that the $\lambda_{i}$ versus $k$ plot for CdTe(111) behaves like the left panel of Fig. 1(b).

As shown in Fig. 3(a), the lattice planes of $\mathrm{Cd}$ and Te are stacked with two alternating interlayer spacings. By choosing a central plane between two neighboring $\mathrm{Cd}$ and $\mathrm{Te}$ atomic planes bonded in the [111] direction as $S$, one can define a planar embedding surface $S$ cutting no MT surfaces of $\mathrm{Cd}$ and Te atoms on both sides of $S$. As a consequence, the basis functions given by Eq. (19) become orthonormal in the present case. Figure 3(b) shows the projected bulk bands of CdTe(111) along the $\Gamma-M$ line, which was calculated by a bulk LAPW code. Each line represents the energy dispersion of a 3D energy band with $2 \mathrm{D} \mathbf{k}$ at a fixed value of $k_{z}$, the $z$ component of the $3 \mathrm{D}$ wave vector, which is varied with a small mesh interval between $\left[-\pi / d_{z}, \pi / d_{z}\right]$.

In Fig. 3(c), we show the embedding-potential eigenvalues of $\mathrm{CdTe}(111)$ along a constant energy path $\epsilon=\epsilon_{1}$ linking $\Gamma$ and $M$ in the projected bulk band gap between the valence and conduction bands shown by a dashed line in panel (b). The cutoff parameter for the basis functions is chosen as $G_{\text {cut }}=3.5$ a.u. As stated in the preceding section, a bunch of eigenvalues with $\tan ^{-1} \lambda_{i} \sim-\tan ^{-1}\left(G_{\text {cut }} / 2\right)=$ -1.05 forming the lower edge of the $\lambda_{i}$ bands correspond to the evanescent waves strongly localized near $S$. Figure 3(d) shows an enlargement of panel (c) corresponding to a small $\lambda$ interval marked with a vertical blue arrow. These eigenstates are well converged and do not change upon increasing $G_{\text {cut }}$. It is seen that two states in a Kramers pair at $\Gamma$ form a Kramers pair again at $M$, and also that each pair of states is separated from neighboring pairs by a $\lambda$ gap. Qualitative features of these lines coincide with those in the left panel of Fig. 1(b), confirming that the topological invariant along $\Gamma-M, \pi_{\Gamma} \pi_{M}$, equals unity.

Here, it may be worth to address a particular property of the systems having $\mathrm{C}_{3 v}$ symmetry. That is, due to the mirror reflection symmetry about the $y z$ plane, generalized Bloch states with $\mathbf{k}$ along the $\Gamma-M$ line are either even or odd with respect to the mirror reflection operation about the $y z$ plane, $\hat{\sigma}_{y z}$ [41]. Assuming that $\phi_{i}$, one of the two states forming a Kramers pair at $\Gamma$ or $M$, is even with respect to $\hat{\sigma}_{y z}$, it is easy to show that the other state in the pair, $\hat{T} \phi_{i}$, is odd with respect to $\hat{\sigma}_{y z}$. This means that a pair of states forming a Kramers doublet at both $\Gamma$ and $M$ have opposite parities with respect to $\hat{\sigma}_{y z}$ along the $\Gamma-M$ line. As a consequence, the two lines forming a pair in Fig. 3(d) can cross each other in the middle of the $\Gamma-M$ line.

\section{D. $\operatorname{GeTe}(111)$}

For the second example we consider GeTe crystallized in the rock-salt structure. We used a cubic lattice constant of $6.02 \AA$ reported in a recent work [42]. Similarly to $\mathrm{CdTe}, \mathrm{GeTe}$ is a nontopological insulator with $3 \mathrm{D} Z_{2}$ invariants, $\left(v_{0} ; v_{1} v_{2} v_{3}\right)=(0 ; 000)$. We calculate the eigenvalues of $\hat{\Sigma}_{L}(k, \epsilon)$ for a semi-infinite $\mathrm{GeTe}(111)$ along the $\Gamma-M$ line. As seen from Fig. 4(a), the system consists of alternating Ge and Te atomic planes, which are stacked in the [111] direction with the same layer spacing and form a hexagonal lattice with lattice constant $a=6.02 / \sqrt{2} \AA$. Since the MT spheres of atoms are nearly as large as touching spheres, the embedding surface $S$ weaving between two neighboring $\mathrm{Ge}$ and $\mathrm{Te}$ atomic planes becomes a curvy surface to avoid overlapping with the MT spheres of atoms on both sides of $S$. In Fig. 4(b), we show the projected bulk bands of $\mathrm{GeTe}(111)$ along the $\Gamma-M$ line.

The eigenvalues $\lambda_{i}(k, \epsilon)$ vary with $k, \epsilon$, and also with the choice of the embedding surface $S$. Here, we illustrate how the eigenvalues change with energy $\epsilon$. In Fig. 4(c), we show the embedding-potential eigenvalues of $\mathrm{GeTe}(111)$ along a constant energy path $\epsilon=\epsilon_{1}$ in the projected band gap between the valence and conduction bands shown by a dashed line in panel (b). Since $\pi_{\Gamma} \pi_{M}=(-1)^{\nu_{0}}=1$, the eigenvalues of $\hat{\Sigma}_{L}(k, \epsilon)$ form pairs of states, which are degenerate both at $\Gamma$ and $M$, and are separated from neighboring pairs by $\lambda$ gaps. Interestingly, in contrast to the left panel of Fig. 1(b), where all the $\lambda_{i}$ values are finite, it is seen that the two states forming a degenerate pair at $\Gamma$ with the largest $\lambda_{i}$ value $\left(\tan ^{-1} \lambda_{i}=1.21\right)$ diverge to $+\infty$ at two poles, $k=k_{1}$ and $k_{2}$, in the middle of the $\Gamma-M$ line. After crossing the poles, they emerge from $\lambda=-\infty$ and disperse with $k$ on the negative $\lambda$ side. Moreover, before reaching the $M$ point, they diverge again to $-\infty$ at two poles, 
(a)
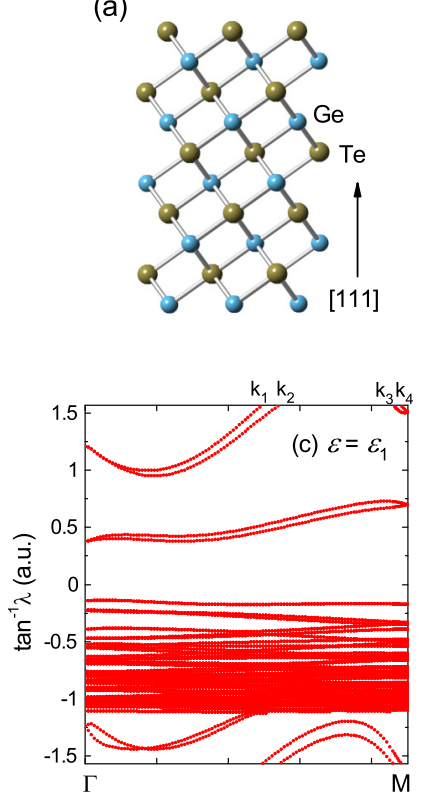

(b)
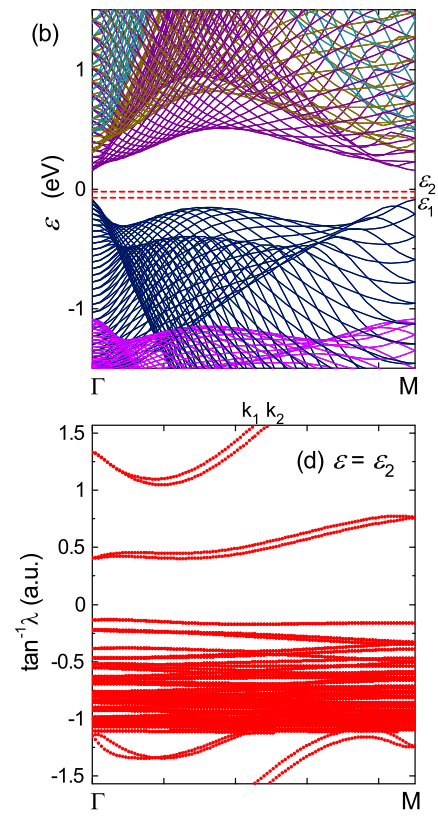

FIG. 4. (a) Crystal structure of GeTe seen from a direction perpendicular to the [111] axis. (b) Projected bulk bands of GeTe(111) along the $\Gamma-M$ line. (c) Eigenvalue spectrum of the embedding potential of $\mathrm{CdTe}(111)$ along a constant energy path $\epsilon=\epsilon_{1}$ shown by a dashed line in (b). (d) The same as (c) for a slightly larger energy $\epsilon=\epsilon_{2}$

$k=k_{3}$ and $k_{4}$, changing their sign, and form a Kramers pair at $M$ on the positive $\lambda$ side with $\tan ^{-1} \lambda_{i}=1.51$. As discussed in Sec. II, the existence of a pole in $\lambda_{i}$ indicates that one can construct an evanescent wave $\phi_{i}$ whose amplitude vanishes identically on $S$ at $(k, \epsilon)$. If one plots $\lambda_{i}^{-1}$ instead of $\lambda_{i}$ as a function of $k$, the two states exhibit no singularity. Rather, the $\lambda_{i}^{-1}$ versus $k$ plot of the two states varies smoothly through $\Gamma$ and $M$, crossing the line $\lambda^{-1}=0$ at $k_{1}$ and $k_{4}$ or at $k_{2}$ and $k_{3}$. It should be also noted that the fact that these two states exhibit large negative $\lambda_{i}$ values in the second half of the $\Gamma-M$ line does not mean that the corresponding eigenfunctions $\phi_{i}$ are strongly localized near $S$, as is the case for a bunch of eigenstates with $\lambda_{i} \sim-G_{\text {cut }} / 2$. Instead, the $\lambda_{i}$ values of this pair take large values, simply because the denominator of the equation $\lambda_{i}=\partial_{n} \phi_{i} /\left(2 \phi_{i}\right)$, which holds on every point on $S$, is much smaller than its numerator.

Figure 4(d) shows the embedding-potential eigenvalues of $\mathrm{GeTe}(111)$ along the $\Gamma-M$ line at a slightly larger energy $\epsilon=\epsilon_{2}$ than $\epsilon_{1}$ in the projected bulk band gap. The eigenvalue spectra in Figs. 4(c) and 4(d) are qualitatively very similar. The two states in panel (d) forming a degenerate pair at $\Gamma$ with the largest $\lambda_{i}$ value diverge to $+\infty$ at two poles $\left(k_{1}\right.$ and $\left.k_{2}\right)$ and change their sign similarly to the corresponding ones in panel (c). However, the two states do not return to the positive $\lambda$ side differently from the corresponding ones in panel (c). Instead, the two states form a Kramers doublet at $M$ on the negative $\lambda$ side with $\tan ^{-1} \lambda_{i}=-1.24$.

\section{E. $\operatorname{Bi}(111)$}

From the results for the empty lattice, CdTe, and GeTe, one may say that the total number of poles that $\lambda_{i}(k, \epsilon)$
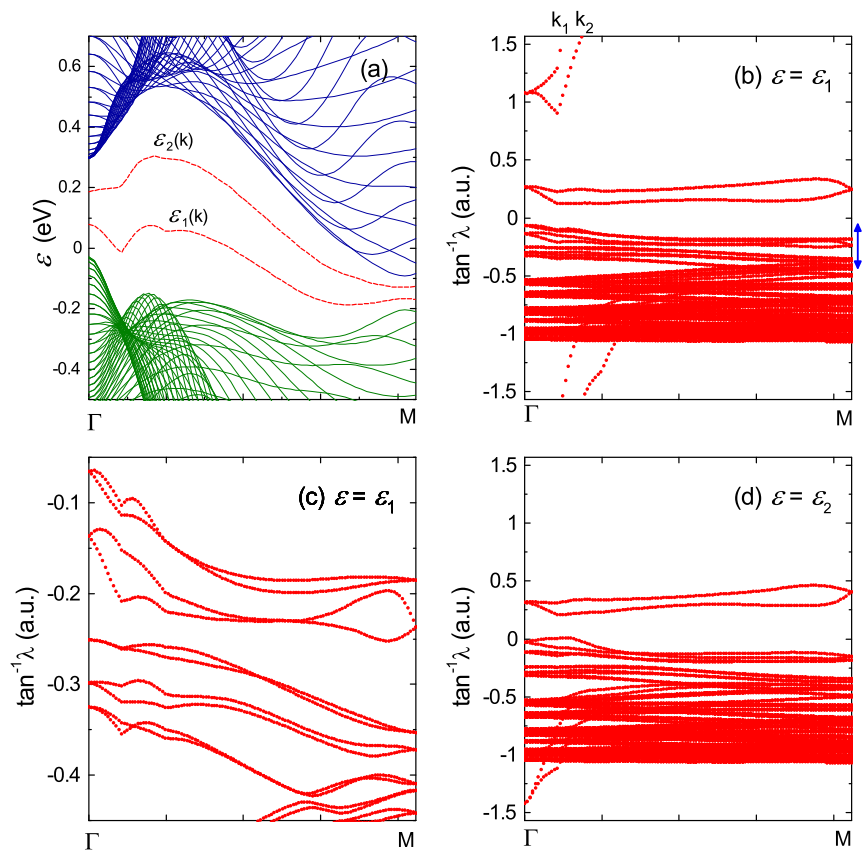

FIG. 5. (a) Projected bulk bands of $\mathrm{Bi}(111)$ along the $\Gamma-M$ line. Blue and green lines correspond to the highest valence band and lowest conduction bands, respectively. (b) Eigenvalue spectrum of the embedding potential of $\mathrm{Bi}(111)$ along energy path $\epsilon=\epsilon_{1}(k)$ shown by a dashed line in (a). (c) Enlargement of (b) corresponding to a small $\lambda$ interval indicated by vertical blue arrow in panel (b). (d) The same as (b) for energy path $\epsilon=\epsilon_{2}(k)$.

$(i=1,2, \ldots, 2 N)$ exhibit along a path linking two TRIM points, $k_{\alpha}$ and $k_{\beta}$, in a projected bulk band gap is even, if the product of time-reversal polarization, $\pi_{\alpha} \pi_{\beta}$ is unity. Because of Eq. (12), one may also say that the number of poles that the embedding potential $\hat{\Sigma}_{L}(k, \epsilon)$ exhibits along the same path is even. Although we considered so far only constantenergy paths, this also holds for any curvy path $\epsilon=\epsilon(k)$ $\left(k_{\alpha} \leqslant k \leqslant k_{\beta}\right)$ passing in a projected bulk band gap from $k_{\alpha}$ to $k_{\beta}$. To illustrate this, we calculate the embedding-potential eigenvalues of $\mathrm{Bi}(111)$.

Bismuth crystallizes in the rhombohedral A7 structure, in which hexagonal 2D layers are stacked perpendicularly to the [111] direction with the interlayer spacing alternating between two values $[43,44]$. A pair of nearest-neighbor layers (bylayer) with shorter layer spacing is covalently bonded, while nearest bylayers with larger layer spacing are more weakly coupled. We choose the embedding surface such that it weaves between two neighboring bylayers.

In Fig. 5(a), we show the projected bulk bands of $\mathrm{Bi}(111)$ along the $\Gamma-M$ line. While $\mathrm{Bi}$ is a semi-metal, its valence bands can be characterized by the $Z_{2}$ topological invariants, since $\mathrm{Bi}$ has a finite direct energy gap throughout the Brillouin zone. $\mathrm{Fu}$ and Kane [20] proposed $\mathrm{Bi}_{1-x} \mathrm{Sb}_{x}$ alloys as a candidate of $3 \mathrm{D}$ topological insulators in a certain range of $x$ values, where $\mathrm{Bi}$, the end component with $x=0$, is a trivial insulator with $\left(v_{0} ; v_{1} v_{2} v_{3}\right)=(0 ; 000)$. For $\mathrm{Bi}$, it is not possible to define a constant energy path passing in the projected bulk band gap from $\Gamma$ to $M$ because the maximum of the projected valence bands is higher in energy than the minimum of the projected 
conduction bands. Instead, we consider two curvy paths $\epsilon=$ $\epsilon_{1,2}(k)$, which trisect the projected bulk band gap between the valence and conduction bands of $\mathrm{Bi}$.

Figure 5(b) shows the calculated embedding-potential eigenvalues of $\operatorname{Bi}(111)$ along $\epsilon=\epsilon_{1}(k)$ in the whole range, while panel (c) is its enlargement corresponding to a small $\lambda$ interval marked with a blue arrow in panel (b). As in the case of CdTe and GeTe, it is seen that the two states in a Kramers pair at $\Gamma$ form a Kramers doublet again at $M$ and each pair of states is separated from neighboring one, which is consistent with the topological characterization of the Bi valence bands. The two states in the Kramers pair with the largest eigenvalue at $\Gamma\left(\tan ^{-1} \lambda_{i}=1.08\right)$ diverge to $+\infty$ at two poles, $k_{1}$ and $k_{2}$, and jump to $\lambda=-\infty$ and then further disperse with $k$ on the negative $\lambda$ side, until they are merged into the densely distributed $\lambda_{i}$ bands with $\lambda_{i} \sim-G_{\text {cut }} / 2$.

Figure $5(\mathrm{~d})$ shows the calculated eigenvalues of $\Sigma_{L}(k, \epsilon)$ along path $\epsilon=\epsilon_{2}(k)$ shown in panel (a). Qualitatively, the eigenvalue spectra are very similar to those in panel (b). As a difference, the embedding-potential exhibits no poles on the path $\epsilon=\epsilon_{2}(k)$. The Kramers pair with $\tan ^{-1} \lambda_{i}=1.08$ in panel (b) shifts to larger $\lambda$ values and change sign at some energy between $\epsilon_{1}\left(k_{\Gamma}\right)$ and $\epsilon_{2}\left(k_{\Gamma}\right)$. As a result, the corresponding Kramers pair at $\Gamma$ appears on the negative $\lambda$ side with $\tan ^{-1} \lambda_{i}=-1.42$.

\section{F. $\mathrm{TlBiSe}_{2}(111)$}

As the first example of topological insulators, we consider $\mathrm{TlBiSe}_{2}$ with $3 \mathrm{D} Z_{2}$ invariants, $\left(v_{0} ; v_{1} v_{2} v_{3}\right)=(1 ; 000)$. At first, this material was shown to be a topological insulator by the parity analyses of the valence-band wave functions within DFT [11]. This prediction was later verified by photoemission experiments in which a 2D Dirac cone crossing the bulk band gap was observed [13-15]. As shown in Fig. 6(a), TlBiSe 2 is composed of hexagonal atomic layers, which are stacked in the surface normal $(z)$ direction in the same sequence as a face centered cubic (FCC) crystal, that is, in the sequence $\mathrm{ABCABC} . .$. , where $\mathrm{A}, \mathrm{B}$, and $\mathrm{C}$ refer to the three atomic sites of the FCC lattice when atoms are projected onto the (111) plane. Regarding atomic arrangement, the lattice planes are stacked in the repetition of the sequence Se-Bi-Se-Tl, so that one unit cell contains four atomic layers, among which $\mathrm{Bi}$ and $\mathrm{Tl}$ atomic sites are the inversion centers. In the present work, we employ the bulk lattice parameters determined by a DFT calculation reported by Singh et al. [45].

We consider a $\mathrm{TlBiSe}_{2}$ crystal oriented in the [111] direction, where we use the rhombohedral unit cell to define crystal orientations. The left half-space is defined by a curvy embedding surface located at the middle of two neighboring Tl and Se layers. We calculate the eigenvalues of $\hat{\Sigma}_{L}(k, \epsilon)$ along the $\Gamma-M$ line. Since $\pi_{\Gamma} \pi_{M}=\pi_{\Gamma}\left(\pi_{M}\right)^{3}=(-1)^{\nu_{0}}=-1$, it is expected that the $\lambda_{i}$ versus $k$ plot of $\mathrm{TlBiSe}_{2}$ behaves like the right panel of Fig. 1(b).

Figures 6(b) and 6(c) show, respectively, the projected bulk bands of $\mathrm{TlBiSe}_{2}(111)$ along the $\Gamma-M$ line and the eigenvalues of $\hat{\Sigma}_{L}(k, \epsilon)$ along a constant energy path $\epsilon=\epsilon_{1}$ in the projected bulk band gap between the valence and conduction bands. The cutoff parameter for the basis functions is chosen as $G_{\text {cut }}=$ 3.5 a.u. As already mentioned, while the lowest $\lambda_{i}$ values (a)
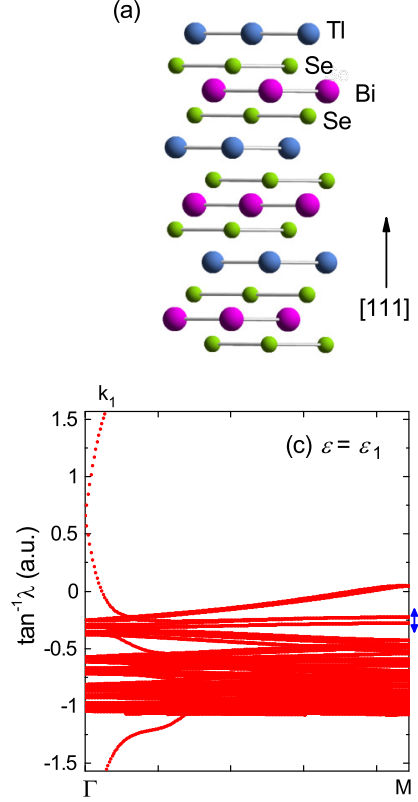

(b)
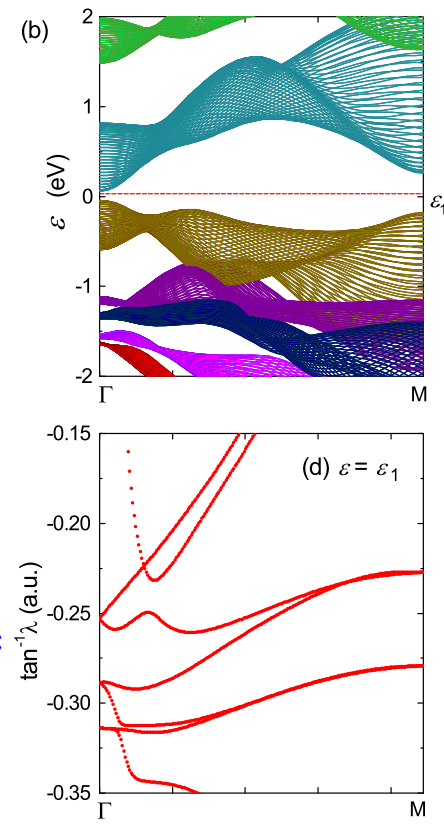

FIG. 6. (a) Crystal structure of $\mathrm{TlBiSe}_{2}$ seen from a direction perpendicular to the [111] axis. (b) Projected bulk bands of TlBiSe along the $\Gamma-M$ line. (c) Eigenvalue spectrum of the embedding potential of $\mathrm{TlBiSe}_{2}(111)$ along a constant energy path $\epsilon=\epsilon_{1}$ shown by a dashed line in (b). (d) Enlargement of (c) corresponding to a small $\lambda$ interval indicated by vertical blue arrow in (c).

corresponding to the evanescent waves strongly localized near $S\left(\lambda_{i} \sim-G_{\text {cut }} / 2\right)$ depend on the cutoff parameter, the other eigenvalues including the zeros and poles of $\hat{\Sigma}_{L}(k, \epsilon)$ are well converged with respect to $G_{\text {cut }}$. It is seen that the eigenvalue spectrum as a function of $k$ exhibits a single pole at $k=k_{1}$. Figure $6(d)$ is an enlargement of panel (c) corresponding to a small $\lambda$ interval marked with a vertical blue arrow. As is seen, in clear contrast to the cases of CdTe and GeTe, two states in a Kramers pair at $\Gamma$ do not form a Kramers pair at $M$. Instead, the upper branch of a Kramers pair at $\Gamma$ and the lower branch of its nearest-neighbor Kramers pair at $\Gamma$ having a larger $\lambda_{i}$ value form a Kramers pair at $M$. As a result, the ensemble of line segments $\lambda=\lambda_{i}(k, \epsilon)(i=1,2, \ldots)$ form a single zigzag line alternating between $\Gamma$ between $M$ from $\lambda=-\infty$ to $+\infty$ with a single pole at $k=k_{1}$. These qualitative features agree fully with those in the right panel of Fig. 2(b), indicating that $\pi_{\Gamma} \pi_{M}=-1$ for the present system.

It should be also noted that the two states forming a Kramers doublet at $\Gamma$ or $M$ have opposite parities with respect to the mirror reflection operation $\hat{\sigma}_{y z}$ due to $\mathrm{C}_{3 v}$ symmetry as mentioned before. As a result, two neighboring line segments, $\lambda=\lambda_{i}\left(k, \epsilon_{1}\right)$ and $\lambda=\lambda_{i+1}\left(k, \epsilon_{1}\right)$, which meet each either at $\Gamma$ or $M$, can cross each other in the middle of the $\Gamma-M$ line. This applies, for example, for the crossing point of two lines at $\tan ^{-1} \lambda \sim-0.225$ in Fig. 6(d).

\section{G. $\mathrm{Bi}_{2} \mathrm{Se}_{3}(\mathbf{1 1 1})$}

As the second example of topological insulators, we consider $\mathrm{Bi}_{2} \mathrm{Se}_{3}$ having the same $3 \mathrm{D} Z_{2}$ invariants $\left(v_{0} ; v_{1} v_{2} v_{3}\right)=$ $(1 ; 000)$ as $\mathrm{TlBiSe}_{2}[7,8]$. As is known, the unit cell of $\mathrm{Bi}_{2} \mathrm{Se}_{3}$ 

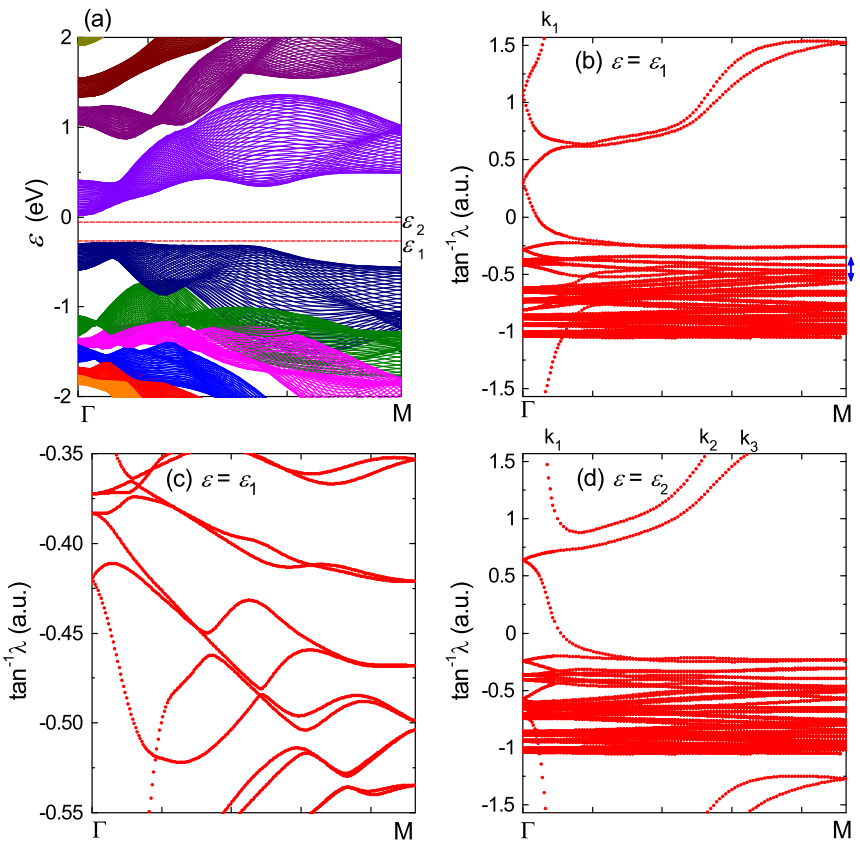

FIG. 7. (a) Projected bulk bands of $\mathrm{Bi}_{2} \mathrm{Se}_{3}(111)$ along the $\Gamma-M$ line. (b) Eigenvalue spectrum of the embedding potential of $\mathrm{Bi}_{2} \mathrm{Se}_{3}(111)$ along a constant energy path $\epsilon=\epsilon_{1}$ shown by a dashed line in (a). (c) Enlargement of panel (b) corresponding to a small $\lambda$ interval indicated by vertical blue arrow in (b). (d) The same as (b) for a larger energy $\epsilon=\epsilon_{2}$.

contains five hexagonal atomic layers stacked in the sequence $\mathrm{Se}-\mathrm{Bi}-\mathrm{Se}-\mathrm{Bi}-\mathrm{Se}$ (quintuple layer) in the [111] direction, among which Se atomic sites in the third layer form space inversion centers. Neighboring atoms within the same quintuple layer are strongly bonded, whereas two neighboring quintuple layers are more weakly bonded, leading to a larger layer spacing between the two neighboring Se layers at the boundary of two quintuple layers than those within the same quintuple layer. The embedding surface $S$ is chosen such that it weaves between these two neighboring Se atomic layers. We employ the lattice parameters reported in the recent experimental work of dos Reis et al. [46].

In Fig. 7(a), we show the calculated projected bulk bands of $\mathrm{Bi}_{2} \mathrm{Se}_{3}(111)$ along the $\Gamma-M$ line. We plot the eigenvalues of $\hat{\Sigma}_{L}(k, \epsilon)$ along two constant energy paths, $\epsilon=\epsilon_{1}$ and $\epsilon=\epsilon_{2}$, passing between $\Gamma$ and $M$ in the projected band gap between the valence and conduction bands. Since $\pi_{\Gamma} \pi_{M}=(-1)^{\nu_{0}}=$ -1 , the $\lambda_{i}$ versus $k$ plot of $\mathrm{Bi}_{2} \mathrm{Se}_{3}(111)$ should behave like the right panel of Fig. 1(b) as in the case of $\mathrm{TlBiSe}_{2}$. In fact, the eigenvalue spectrum for $\epsilon=\epsilon_{1}$ shown in panel (b) exhibits a single pole at $k=k_{1}$. Figure 7(c) is an enlargement of panel (b) corresponding to a small $\lambda$ interval marked with a vertical blue arrow. It is clearly seen that two states in a Kramers pair at $\Gamma$ change their partners at $M$. As a result, the set of line segments $\lambda=\lambda_{i}(k, \epsilon)(i=1,2, \ldots)$ forms a single connected line, alternating between $\Gamma$ and $M$. It is also seen that two neighboring line segments, which become degenerate at $\Gamma$ or $M$, cross each other in the middle of the $\Gamma-M$ line because they have opposite parities with respect to the mirror reflection $\hat{\sigma}_{y z}$.
Furthermore, similarly to the previous example of GeTe and $\mathrm{Bi}(111)$, the number of poles along the $\Gamma-M$ line changes by multiples of 2 depending on the energy value in the projected bulk band gap. In Fig. 7(d), we plot the eigenvalues of $\hat{\Sigma}_{L}(k, \epsilon)$ at a larger energy $\epsilon=\epsilon_{2}$, for which one finds three poles along the $\Gamma-M$ line. Looking into more details, one sees that the two states forming a Kramers pair at $M$ with the largest $\lambda_{i}$ value $\left(\tan ^{-1} \lambda_{i}=1.53\right.$ at $\left.\epsilon=\epsilon_{1}\right)$ shift upward with increasing $\epsilon$. As a result, at $\epsilon=\epsilon_{2}$, the two states diverge to $+\infty$ at two poles, $k=k_{2}$ and $k_{3}$. After crossing these poles, they emerge from $\lambda=-\infty$ and form a Kramers pair at $M$ on the negative $\lambda$ side with $\tan ^{-1} \lambda_{i}=-1.27$. Similarly, the Kramers pair at $\Gamma$ with the largest positive $\lambda_{i}$ value $\left(\tan ^{-1} \lambda_{i}=1.07\right.$ at $\left.\epsilon=\epsilon_{1}\right)$ shifts upward with increasing $\epsilon$, transferring to the negative $\lambda$ side at $\epsilon=\epsilon_{2}$ (its position is hard to see, as it overlaps and interacts with the dense distribution of $\lambda_{i}$ bands with $\lambda_{i} \geqslant-G_{\text {cut }} / 2$ ). As a result, the residue of the pole at $k=k_{1}$ changes sign between $\epsilon_{1}$ and $\epsilon_{2}$.

From the results for $\mathrm{TlBiSe} \mathrm{B}_{2}$ and $\mathrm{Bi}_{2} \mathrm{Se}_{3}$, we confirm that the total number of poles that the embedding potential exhibits along a path linking two TRIM points, $k_{\alpha}$ and $k_{\beta}$, in a projected bulk band gap is $o d d$, if $\pi_{\alpha} \pi_{\beta}=-1$. By denoting this number by $P_{L}$, we find

$$
\pi_{\alpha} \pi_{\beta}=(-1)^{P_{L}},
$$

which holds for both topologically trivial and nontrivial cases. Equivalently, the $Z_{2}$ topological invariant can be expressed as $v=P_{L} \bmod 2$. One may further generalize this relation as $v=N_{L}\left(\lambda_{0}\right) \bmod 2$, where $N_{L}\left(\lambda_{0}\right)$ denotes the number of points at which the line segments $\lambda=\lambda_{i}\left(k, \epsilon_{0}(k)\right)(i=1,2, \ldots)$ intersect $\lambda=\lambda_{0}$ along a path $\epsilon=\epsilon_{0}(k)$ passing in the projected bulk band gap from $k_{\alpha}$ to $k_{\beta}$. Obviously, $P_{L}$ corresponds to $N_{L}\left(\lambda_{0}\right)$ in the limit of $\lambda_{0} \rightarrow \pm \infty$. This is analogous to the well-known criterion for topological insulators that the number of points at which the energy dispersion curves of surface bands with $k$ intersect the Fermi energy is odd $[19,20]$. Both criteria are closely related, since, as discussed in Sec. IIE, they are properties of the lines of intersection when one cuts the $2 \mathrm{D}$ surfaces $\lambda=\lambda_{i}(k, \epsilon)(i=1,2, \ldots)$ in the 3D $(k, \epsilon, \lambda)$ coordinate space by either a vertical or a horizontal plane.

\section{H. Relation to previous works}

It may be useful to discuss the relation between the present method and previous ones for the determination of the $Z_{2}$ invariant of band insulators. Specifically, we will focus on the formulation in which one makes use of the winding number of the Wannier function centers for the occupied valence bands [24,25]. The figures in the paper of Yu et al. [25] showing the evolution of the Wannier function centers along a path connecting two TRIM points might look similar to Figs. 3 to 7 in the present paper. We consider that both methods are not directly related. Our reasoning is as follows.

The Wannier functions in Refs. [24,25] are constructed from the wave functions of the occupied valence bands. Main quantity in the present theory is the embedding potential at an arbitrary energy within the projected band gap between the valence and conduction bands. The embedding potential is constructed from the evanescent waves satisfying the generalized Bloch condition. The character of the wave 
functions of these evanescent waves changes continuously with energy. When the energy is slightly above the upper edge of the projected valence bands, $\epsilon_{v}(k)$, their orbital character is similar to that of the valence bands. On the other hand, when the energy is slightly below the lower edge of the projected conduction bands, $\epsilon_{c}(k)$, their orbital character is similar to that of the conduction bands. Namely, the embedding potential is not a property of the valence bands alone. In other words, if the Hamiltonian matrix is constructed by using the Wannier functions as the basis set, one would need not only the Wannier functions of the valence bands but also those of the conduction bands in order to be able to describe the evanescent waves within the band gap. Thus we may say that, while the Wannier center method derives the $Z_{2}$ topological invariant from the subspace of the Hamiltonian spanned by the occupied valence bands, the present method uses a wider eigenstate space to find the same quantity. In addition, it should be noted that the embedding-potential eigenvalue is a real quantity simply giving the ratio between the amplitude of the evanescent waves and their normal derivative on the boundary surface and has no relation with the eigenvalues of the projected position operator used in the Wannier center method. Also, the number of lines plotted in Figs. 3 to 7 in the present paper increases with the increasing cutoff energy for the basis functions (19) and is different from the number of the occupied bands.

The present method may rather be regarded as a technique to calculate surface states from a single bulk unit-cell calculation without making any computationally more demanding slab or semi-infinite surface calculations. The point is that the existence of gapless surface states for topological band insulators is protected by time-reversal symmetry for any surface boundary condition irrespective of whether the boundary condtion is realistic or only mathematical. As discussed in Sec. IIE, the eigenfunction of the embedding potential in the band gap is a surface state satisfying the mathematical boundary condition (18) on the boundary surface $S$. Hence it may be rather natural that one can predict the emergence of a gapless surface band from the behaviors of the eigenvalues of the embedding potential. Indeed, the poles that the embedding potential $\hat{\Sigma}_{L}(\mathbf{k}, \epsilon)$ exhibits along a path $\epsilon=\epsilon_{0}(\mathbf{k})$ between two TRIM points in the projected bulk band gap are identical with the points at which the energy dispersion curves of the surface bands, which fulfill the particular boundary condition, $\phi(\xi)=0$ on $S$, intersect the line $\epsilon=\epsilon_{0}(\mathbf{k})$ in the energy gap. Hence $P_{L}$ gives the number of points of intersection between $\epsilon=\epsilon_{0}(\mathbf{k})$ and the energy dispersion curves of these bands.

\section{DISCUSSION ON INTERFACE STATES}

In Sec. II E, we discussed the surface states of a semi-infinite crystal occupying the half space to the left of surface $S$ by imposing a mathematical boundary condition, Eq. (18), on the surface-state wave functions. Here, we consider an arbitrary interface between two semi-infinite crystals and discuss under what conditions the localized interface states may occur in the projected bulk band gap of the two crystals. We would like to explain from a view point of the embedding theory why metallic interface bands crossing the bulk band gap occur without exception when one system is a trivial insulator and the other is a topological insulator.
We assume that the semi-infinite crystal on the left-hand side of the interface, $C_{L}$, and that on the right-hand side, $C_{R}$, both are made out of lattice planes stacked in the surface normal $(z)$ direction and have the same 2D unit cell specified by two lattice vectors, $\left\{\mathbf{a}_{1}, \mathbf{a}_{2}\right\}$. Although the atoms near the interface may deviate from the ideal positions due to layer relaxations and restructuring, we assume that the interface holds the $1 \times 1$ structure in the plane. We define an embedding surface $S$ at the interface between the two crystals. To calculate the embedding potential for the left half-space, $\hat{\Sigma}_{L}(\mathbf{k}, \epsilon)$, one starts with the $2 N$ generalized Bloch states, $\psi_{i}$, satisfying the generalized Bloch condition (5) in the interior of $C_{L}$. By integrating the Schrödinger equation with respect to $z$ from the interior of $C_{L}$ through the interface region up to $S$, one obtains the value and normal derivative of these $2 N$ solutions on $S$. Then, $\hat{\Sigma}_{L}(\mathbf{k}, \epsilon)$ is calculated by using Eq. (7). The embedding potential for the right-half space, $\hat{\Sigma}_{R}(\mathbf{k}, \epsilon)$, is calculated in a similar manner from the $2 N$ generalized Bloch states satisfying the outgoing boundary condition at $z=+\infty$ in the interior of $C_{R}$.

Given a 2D wave vector $\mathbf{k}$, localized interface states may occur when the energy $\epsilon$ is located in the projected band gap of $C_{L}$ and in the corresponding one of $C_{R}$ both. The lower and upper boundaries of the intersection of the projected bulk band gaps of the two materials will be denoted by $\epsilon_{v}(\mathbf{k})$ and $\epsilon_{c}(\mathbf{k})$ in the following. Let us denote the wave function of an interface state with $\epsilon$ and $\mathbf{k}$ by $\phi$. Since $\phi$ decays toward the interior of $C_{L}$, its left normal derivative on $S$ is given by Eq. (1). Further, since $\phi$ also decays toward the interior of $C_{R}$, its right normal derivative is given by a similar equation in which $\hat{\Sigma}_{L}$ in Eq. (1) is replaced by $\hat{\Sigma}_{R}$ (note that the surface normal $n$ points to the interior of the half space, so that $n$ for $\hat{\Sigma}_{R}$ is opposite to $n$ for $\hat{\Sigma}_{L}$ ). Since the left and right normal derivatives must be the same, we have on $S$,

$$
\int_{S} d \xi^{\prime} \Sigma\left(\mathbf{k}, \epsilon, \xi, \xi^{\prime}\right) \phi\left(\xi^{\prime}\right)=0
$$

with $\hat{\Sigma}(\mathbf{k}, \epsilon)$ defined by

$$
\hat{\Sigma}(\mathbf{k}, \epsilon) \equiv \hat{\Sigma}_{L}(\mathbf{k}, \epsilon)+\hat{\Sigma}_{R}(\mathbf{k}, \epsilon) .
$$

Equation (25) indicates that the energies of the interface states with $2 \mathrm{D}$ wave vector $\mathbf{k}$ are determined by the equation,

$$
\operatorname{det}[\hat{\Sigma}(\mathbf{k}, \epsilon)]=0 .
$$

As discussed in Sec. IIC, $\hat{\Sigma}_{L}(\mathbf{k}, \epsilon)$ and $\hat{\Sigma}_{R}(\mathbf{k}, \epsilon)$ are Hermitian in the projected bulk band gap of each material, so that $\hat{\Sigma}(\mathbf{k}, \epsilon)$ is Hermitian if $\epsilon_{v}(\mathbf{k})<\epsilon<\epsilon_{c}(\mathbf{k})$. In this energy range, the eigenvalues of $\hat{\Sigma}(\mathbf{k}, \epsilon)$ are real and the corresponding eigenfunctions form an orthonormal basis set on $S$. Thus, similarly to Eqs. (12) and (13), we have

$$
\begin{gathered}
\hat{\Sigma}(\mathbf{k}, \epsilon)=\sum_{i} \lambda_{i}(\mathbf{k}, \epsilon)\left|\phi_{i}\right\rangle\left\langle\phi_{i}\right|, \\
\operatorname{det}[\hat{\Sigma}(\mathbf{k}, \epsilon)]=\prod_{i} \lambda_{i}(\mathbf{k}, \epsilon),
\end{gathered}
$$

where $\lambda_{i}$ and $\phi_{i}$ denotes the $i$-th eigenvalue and eigenfunction of $\hat{\Sigma}(\mathbf{k}, \epsilon)$. From Eqs. (27) and (29), we see that the energies of the interface state are given by the zeros of the eigenvalues $\lambda_{i}(\mathbf{k}, \epsilon)$. 
Now, we assume that the system in consideration has timereversal symmetry. Due to Eq. (16) and the corresponding one for $\hat{\Sigma}_{R}$, we have

$$
\hat{T} \hat{\Sigma}(\mathbf{k}, \epsilon) \hat{T}^{-1}=\hat{\Sigma}(-\mathbf{k}, \epsilon),
$$

if $\epsilon_{v}(\mathbf{k})<\epsilon<\epsilon_{c}(\mathbf{k})$. From this equation, we will see that $\hat{T} \phi_{i}$, with $\phi_{i}$ being the eigenfunction of $\hat{\Sigma}(\mathbf{k}, \epsilon)$ with eigenvalue $\lambda_{i}(\mathbf{k}, \epsilon)$, is an eigenfunction of $\hat{\Sigma}(-\mathbf{k}, \epsilon)$ with the same eigenvalue. Therefore, similarly to Eq. (15), we have

$$
\lambda_{i}(-\mathbf{k}, \epsilon)=\lambda_{i}(\mathbf{k}, \epsilon) .
$$

In what follows, we assume that $\mathbf{k}$ is along a path connecting two TRIM points, $\mathbf{k}_{\alpha}$ and $\mathbf{k}_{\beta}(\alpha, \beta=\mathrm{I} \sim \mathrm{IV})$. We use a scalar variable $k$ to represent $2 \mathrm{D}$ wave vector $\mathbf{k}$ on this path. As we did in Sec. II D, it is useful to plot 2D surface $\lambda=\lambda_{i}(k, \epsilon)$ in the 3D Cartesian coordinate system having the horizontal $k \epsilon$ plane and the vertical $\lambda$ axis [see Fig. 1(a)]. Let us consider the lines of intersection between surfaces $\lambda=\lambda_{i}(k, \epsilon)(i=1,2, \ldots)$ and a vertical cut plane $\epsilon=\epsilon_{0}$ in the projected bulk band gap. Then, these lines, $\lambda=\lambda_{i}\left(k, \epsilon_{0}\right)(i=1,2, \ldots)$, will behave like either the left or right panel of Fig. 1(b), since they form Kramers doublets at both end points, $k_{\alpha}$ and $k_{\beta}$, because of Eq. (31). In the present case, the energy dispersion relations with $k$ of the interface states are given by the lines of intersection between surfaces $\lambda=\lambda_{i}(k, \epsilon)(i=1,2, \ldots)$ and the horizontal cut plane $\lambda=0$ as mentioned in the above. Following the same arguments in Sec. II E, we may conclude that interface bands crossing the projected bulk band gap inevitably occur if the set of lines $\lambda=\lambda_{i}\left(k, \epsilon_{0}\right)(i=1,2, \ldots)$ behave like the right panel of Fig. 1(b). As discussed in Sec. III, the behaviors of these lines are related with the number of poles that these lines exhibit or equivalently with the number of poles that $\hat{\Sigma}\left(k, \epsilon_{0}\right)$ exhibits along the path linking $k_{\alpha}$ and $k_{\beta}$. The lines of intersection behave like the right panel of Fig. 1(b) if this number is odd. Because of the definition Eq. (26), the poles of $\hat{\Sigma}\left(k, \epsilon_{0}\right)$ arise either from the poles of $\hat{\Sigma}_{L}\left(k, \epsilon_{0}\right)$ or those of $\hat{\Sigma}_{R}\left(k, \epsilon_{0}\right)$. Namely, if the numbers of these poles are denoted by $P, P_{L}$, and $P_{R}$, respectively, we will have $P=P_{L}+P_{R}$. This indicates that surface states crossing the bulk band gap inevitably occur for two combinations, $\left(P_{L}, P_{R}\right)=($ even, odd $)$ and (odd, even). In the case of crystal surfaces, the semi-infinite vacuum on the right-hand side may be regarded as an empty lattice with $P_{R}=0$. Thus the surface bands crossing the projected bulk band gap always appear if $P_{L}$ is odd.

Before closing, one comment is in order. In the end of Sec. II B, we emphasized that the embedding potential is a bulk quantity obtained from generalized Bloch states. The embedding potentials, $\hat{\Sigma}_{L}$ and $\hat{\Sigma}_{R}$, in this section are different in the sense that, in order to determine them, one needs to calculate the value and normal derivative of the generalized Bloch states on $S$ by integrating the Schrödinger equation through the interface region where the potential may deviate from the bulk one. In this case, the eigenvalues of the embedding potential may vary continuously, as the embedding surface $S$ is gradually moved from the interior of the crystal toward the interface region. While the number of poles may vary by a multiple of 2 similarly to Figs. 4,5 , and 7, where energy $\epsilon$ was changed rather than the position of $S$, it is unlikely that the behaviors of the embedding-potential eigenvalues switch suddenly from those in the left panel of Fig. 1(b) to those in the right panel or vice versa. Therefore we believe that $P_{L}$ and $P_{R} \bmod 2$ are bulk properties.

\section{SUMMARY AND CONCLUSION}

The embedding technique invented by Inglesfield [27,28] has been successfully applied to the electronic structure calculation of inhomogeneous systems such as surfaces and interfaces. The embedding potential of a semi-infinite crystal relates the value and normal derivative on embedding surface $S$ of generalized Bloch states with energy $\epsilon$ and 2D wave vector $\mathbf{k}$ propagating or decaying toward the interior of the crystal.

In the present work, we have shown that the embedding potential of a time-reversal invariant band insulator for a given crystal orientation can be used to determine the $Z_{2}$ topological invariants of the material associated with the crystal orientation. For this purpose, one only has to plot real eigenvalues of the embedding potential in the interior of the projected bulk band gap along an arbitrary path $\epsilon=\epsilon_{0}(\mathbf{k})$ connecting two TRIM points in the SBZ, $\mathbf{k}_{\alpha}$ and $\mathbf{k}_{\beta}$. They form Kramers pairs at both TRIM points, and from the two distinct ways how they behave along the path, one easily finds the $Z_{2}$ invariant associated with the two TRIM points, $\pi_{\alpha} \pi_{\beta}=(-1)^{\nu}$, where $\pi_{\alpha}$ and $\pi_{\beta}$ denote the time-reversal polarizations at the two points. Furthermore, we have derived a simplified equation, $v=P \bmod 2$, where $P$ denotes the number of poles that the embedding potential exhibits along the path $\epsilon=\epsilon_{0}(\mathbf{k})$ connecting the two TRIM points. To determine all the independent $Z_{2}$ invariants for 3D band insulators, one would need to calculate the real eigenvalues of the embedding potential in the projected bulk band gap for more than one crystal orientations depending on the symmetry of a given system.

As an application, we calculated real eigenvalues of the embedding potential in the projected bulk band gap between the valence and conduction bands for the (111) surfaces of $\mathrm{CdTe}, \mathrm{GeTe}, \mathrm{Bi}, \mathrm{BiTlSe} e_{2}$, and $\mathrm{Bi}_{2} \mathrm{Se}_{3}$ within DFT. For the former three systems, the number of poles along the path connecting the $\Gamma$ and $M$ points was even, while the corresponding one for the latter two insulators was odd in full accord with the topological classification of the five materials.

We have also discussed, from the embedding view point, under what conditions localized interface states crossing the bulk band gap occur at the interface between two band insulators. It was shown that such gapless band inevitably emerges if the sum of the number of the poles that the embedding potential for the left-half system exhibits along the path connecting two TRIM points and the corresponding one for the right-half system is an odd integer. This occurs only when one of the insulators constituting the interface is a trivial insulator and the other is a topological insulator.

\section{ACKNOWLEDGMENTS}

H.I. gratefully acknowledges funding under MEXT KAKENHI No. 25110006. H.I. is also grateful to Forschungszentrum Jülich for support during his stay in Jülich. 
[1] M. Z. Hasan and C. L. Kane, Rev. Mod. Phys. 82, 3045 (2010).

[2] X. L. Qi and S. C. Zhang, Rev. Mod. Phys. 83, 1057 (2011).

[3] B. Yan and S. C. Zhang, Rep. Prog. Phys. 75, 096501 (2012).

[4] H. Zhang and S. C. Zhang, Phys. Status Solidi RRL 7, 72 (2013).

[5] C. L. Kane and E. J. Mele, Phys. Rev. Lett. 95, 226801 (2005).

[6] B. A. Bernevig, T. L. Hughes, and S. C. Zhang, Science 314, 1757 (2006).

[7] H. Zhang, C. X. Liu, X. L. Qi, X. Dai, Z. Fang, and S. C. Zhang, Nat. Phys. 5, 438 (2009).

[8] W. Zhang, R. Yu, H. Zhang, X. Dai, and Z. Fang, New J. Phys 12, 065013 (2010).

[9] Y. Xia, D. Qian, D. Hsieh, L. Wray, A. Pal, H. Lin, A. Bansil, D. Grauer, Y. S. Hor, R. J. Cava, and M. Z. Hasan, Nat. Phys. 5, 398 (2009).

[10] K. Kuroda, M. Arita, K. Miyamoto, M. Ye, J. Jiang, A. Kimura, E. E. Krasovskii, E. V. Chulkov, H. Iwasawa, T. Okuda, K. Shimada, Y. Ueda, H. Namatame, and M. Taniguchi, Phys. Rev. Lett. 105, 076802 (2010).

[11] B. Yan, C. X. Liu, H. J. Zhang, C. Y. Yam, X. L. Qi, T. Frauenheim, and S. C. Zhang, Europhys. Lett. 90, 37002 (2010).

[12] H. Lin, R. S. Markiewicz, L. A. Wray, L. Fu, M. Z. Hasan, and A. Bansil, Phys. Rev. Lett. 105, 036404 (2010).

[13] T. Sato, K. Segawa, H. Guo, K. Sugawara, S. Souma, T. Takahashi, and Y. Ando, Phys. Rev. Lett. 105, 136802 (2010).

[14] K. Kuroda, M. Ye, A. Kimura, S. V. Eremeev, E. E. Krasovskii, E. V. Chulkov, Y. Ueda, K. Miyamoto, T. Okuda, K. Shimada, H. Namatame, and M. Taniguchi, Phys. Rev. Lett. 105, 146801 (2010).

[15] Y. L. Chen, Z. K. Liu, J. G. Analytis, J. H. Chu, H. J. Zhang, B. H. Yan, S. K. Mo, R. G. Moore, D. H. Lu, I. R. Fisher, S. C. Zhang, Z. Hussain, and Z.-X. Shen, Phys. Rev. Lett. 105, 266401 (2010).

[16] C. L. Kane and E. J. Mele, Phys. Rev. Lett. 95, 146802 (2005).

[17] L. Fu and C. L. Kane, Phys. Rev. B 74, 195312 (2006).

[18] J. E. Moore and L. Balents, Phys. Rev. B 75, 121306(R) (2007).

[19] L. Fu, C. L. Kane, and E. J. Mele, Phys. Rev. Lett. 98, 106803 (2007).

[20] L. Fu and C. L. Kane, Phys. Rev. B 76, 045302 (2007).

[21] J. C. Y. Teo, L. Fu, and C. L. Kane, Phys. Rev. B 78, 045426 (2008).

[22] T. Fukui and Y. Hatsugai, J. Phys. Soc. Jpn. 76, 053702 (2007).

[23] A. A. Soluyanov and D. Vanderbilt, Phys. Rev. B 83, 035108 (2011).
[24] A. A. Soluyanov and D. Vanderbilt, Phys. Rev. B 83, 235401 (2011).

[25] R. Yu, X. L. Qi, A. Bernevig, Z. Fang, and X. Dai, Phys. Rev. B 84, 075119 (2011).

[26] H. Weng, X. Dai, and Z. Fang, MRS Bull. 39, 849 (2014).

[27] J. E. Inglesfield, J. Phys. C 14, 3795 (1981).

[28] J. E. Inglesfield, The Embedding Methodfor Electronic Structure (IOP, Bristol, 2015).

[29] J. E. Inglesfield, Comput. Phys. Commun. 137, 89 (2001).

[30] M. I. Trioni, G. P. Brivio, S. Crampin, and J. E. Inglesfield, Phys. Rev. B 53, 8052 (1996).

[31] M. James and S. Crampin, Phys. Rev. B 81, 155439 (2010).

[32] D. Wortmann, H. Ishida, and S. Blügel, Phys. Rev. B 65, 165103 (2002).

[33] D. Wortmann, H. Ishida, and S. Blügel, Phys. Rev. B 66, 075113 (2002).

[34] H. Ishida, Phys. Rev. B 63, 165409 (2001).

[35] H. Ishida, Phys. Rev. B 90, 235422 (2014).

[36] J. E. Inglesfield, S. Crampin, and H. Ishida, Phys. Rev. B 71, 155120 (2005).

[37] D. J. Singh and L. Nordstrom, Planewaves, Pseudopotentials, and the LAPW Method, 2nd ed. (Springer, Berlin, 2006).

[38] J. P. Perdew, K. Burke, and M. Ernzerhof, Phys. Rev. Lett. 77, 3865 (1996).

[39] F. Freimuth, D. Wortmann, and S. Blügel (unpublished).

[40] M. Bukala, P. Sankowski, R. Buczko, and P. Kacman, Nanoscale Res. Lett. 6, 126 (2011).

[41] To be more accurate, the eigenvalues of $\hat{\sigma}_{y z}$ are $\pm i$ rather than \pm 1 since $\hat{\sigma}_{y z}$ involves rotation in spin space by $\pi$ radians. However, for convenience, the states with eigenvalues $+i$ and $-i$ are called even and odd states, respectively.

[42] K. Hoang, S. D. Mahanti, and M. G. Kanatzidis, Phys. Rev. B 81, 115106 (2010).

[43] P. Cucka and C. S. Barrett, Acta Crystallogr. 15, 865 (1962).

[44] H. Mönig, J. Sun, Yu. M. Koroteev, G. Bihlmayer, J. Wells, E. V. Chulkov, K. Pohl, and Ph. Hofmann, Phys. Rev. B 72, 085410 (2005).

[45] B. Singh, A. Sharma, H. Lin, M. Z. Hasan, R. Prasad, and A. Bansil, Phys. Rev. B 86, 115208 (2012).

[46] D. D. dos Reis, L. Barreto, M. Bianchi, G. A. S. Ribeiro, E. A. Soares, W. S. e Silva, V. E. de Carvalho, J. Rawle, M. Hoesch, C. Nicklin, W. P. Fernandes, J. Mi, B. B. Iversen, and P. Hofmann, Phys. Rev. B 88, 041404(R) (2013). 